\title{
Psychophysical measures of detection \& discrimination and effects of GABA blockade in the moth Manduca sexta
}

\author{
Esther K. Mwilaria \\ West Virginia University
}

Follow this and additional works at: https://researchrepository.wvu.edu/etd

\footnotetext{
Recommended Citation

Mwilaria, Esther K., "Psychophysical measures of detection \& discrimination and effects of GABA blockade in the moth Manduca sexta" (2007). Graduate Theses, Dissertations, and Problem Reports. 4322.

https://researchrepository.wvu.edu/etd/4322

This Thesis is protected by copyright and/or related rights. It has been brought to you by the The Research Repository @ WVU with permission from the rights-holder(s). You are free to use this Thesis in any way that is permitted by the copyright and related rights legislation that applies to your use. For other uses you must obtain permission from the rights-holder(s) directly, unless additional rights are indicated by a Creative Commons license in the record and/ or on the work itself. This Thesis has been accepted for inclusion in WVU Graduate Theses, Dissertations, and Problem Reports collection by an authorized administrator of The Research Repository @ WVU. For more information, please contact researchrepository@mail.wvu.edu.
} 
Psychophysical measures of detection \& discrimination and effects of GABA blockade in the moth Manduca sexta

\author{
By \\ Esther K. Mwilaria \\ Thesis submitted to Eberly College of Arts and Sciences \\ at West Virginia University \\ in partial fulfillment of the requirements \\ for the degree of \\ Master of Science \\ in \\ Biology
}

Approved by

Kevin C. Daly PhD., Committee Chairperson

Christy Foran PhD.

Sarah Farris PhD.

Jorge A. Flores PhD.

Department of Biology

Morgantown, West Virginia

2007

Keywords: antennal lobe, electromyography, odor detection, odor discrimination, olfactory learning, stimulus generalization, threshold 


\title{
Psychophysical measures of detection \& discrimination and effects of GABA blockade in the
}

\author{
moth Manduca Sexta
}

By Esther K. Mwilaria

\begin{abstract}
Olfactory systems must detect and discriminate odors while maintaining a degree of perceptual invariance of identity in a highly variable environment. Studies have suggested that olfactory systems of both vertebrates and invertebrates detect odors based on the interaction of specific molecular features of odorant molecules with olfactory receptor neurons. It has also been shown that changing stimulus concentration can elicit substantive changes in spatial representations of odorants. The first goal of our studies was to psychophysically characterize the ability of the moth Manduca sexta to detect and discriminate odors as a function of concentration using a Pavlovian olfactory-learning paradigm. Our results indicate that odor detection thresholds varied widely with different odors and that discrimination between odors occurred at one or more orders of magnitude above detection. Counterbalancing the reinforced odors produced asymmetric discrimination thresholds which were predictable by the degree of asymmetry in the detection thresholds. Previous studies have also shown that blockade of GABAergic transmission within the insect antennal lobe disrupts discrimination of monomolecular odors with similar molecular features. These studies used generalization protocols, which in principle could falsely imply that discrimination of molecularly different odors is unaffected. Using Manduca sexta and Pavlovianbased stimulus generalization protocols, we confirm that $\mathrm{GABA}_{\mathrm{A}}$ blockade within the antennal lobe increases generalization of a conditioned response to only similar odors as previously demonstrated. However, using differential conditioning protocols, we show that $\mathrm{GABA}_{\mathrm{A}}$ blockade disrupts discrimination of similar and dissimilar odors equally as indicated by increased discrimination thresholds. We also observed an effect on concentration-response functions, suggesting odor detection was also effected. To establish whether disruption of discrimination thresholds could be attributed to disrupted detection thresholds, we investigated the effect of $\mathrm{GABA}_{\mathrm{A}}$ blockade on detection threshold measures. Results show that detection thresholds increased, indicating that disrupted discrimination is due to impairment of the ability to detect; because physiological measures of antennal lobe output indicate that $\mathrm{GABA}_{\mathrm{A}}$ blockade increases output from the antennal lobe, we conclude that our effects are attributable to a loss of ability to extract the neural signal from background activity.
\end{abstract}




\section{DEDICATION}

This work is dedicated to my loving dad the Late Elijah Mwilaria in his memory, my loving mum Beatrice Mwilaria for her hard work and efforts that have made me who I am, as well as my lifetime partner Elijah Mbui for his unwavering support during this process. 


\section{ACKNOWLEDGMENTS}

The author wishes to thank her academic advisor Dr. Kevin C. Daly for his assistance and guidance in this work, Dr. Christy Foran and Dr. Sarah Farris for their advice, guidance and comments, Lynnsey A. Carrell for her collaborative work, Chitrita Ghatak and Herbert Parsons for assistance in data collection as well as Thomas A. Christensen and John G. Hildebrand for generously supplying moths for this research. This work was supported by an award from NIHNIDCD (DC05535-03) to KCD, NIH-NCRR (RR015574-06) to KCD. 


\section{TABLE OF CONTENTS}

Abstract........................................................................

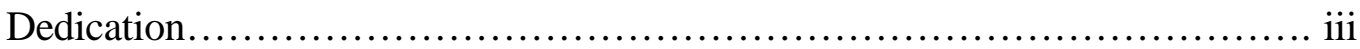

Acknowledgements....................................................iv

Table of contents..........................................................

List of Symbols / Nomenclature..$\ldots \ldots \ldots \ldots \ldots \ldots \ldots \ldots \ldots \ldots \ldots \ldots \ldots \ldots \ldots \ldots$ vi

Introduction.............................................................

Chapter 1 Psychophysical measures of odor detection.................... 6

1. Introduction....................................................... 6

2. Material and Methods............................................... 7

3. Results..........................................................

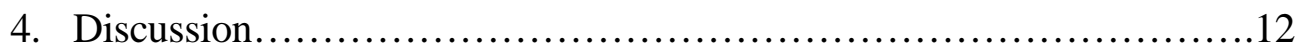

Chapter 2 The effect of intensity on discrimination learning and performance....13

1. Introduction.........................................................

2. Material and Methods.............................................. 14

3. Results..........................................................

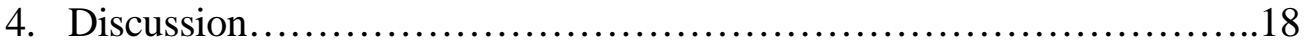

Chapter 3 The effect of GABA blockade on psychophysical measures of odor detection and discrimination........................................ 20

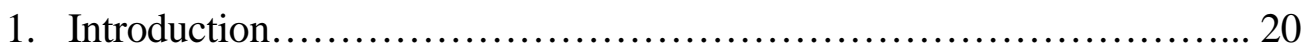

2. Material and Methods.............................................. 21

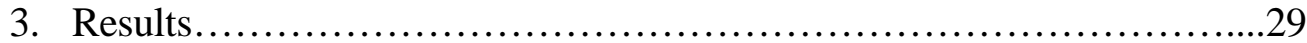

4. Discussion......................................................

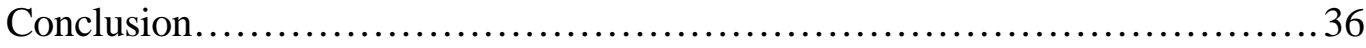

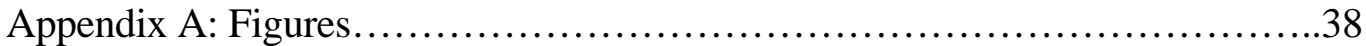

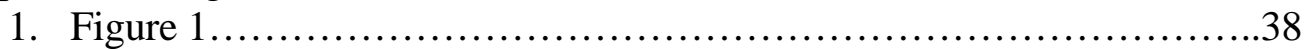

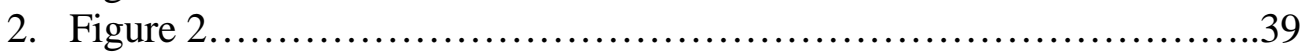

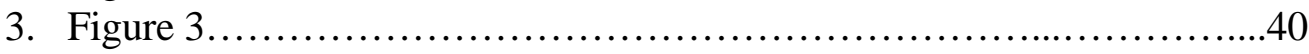

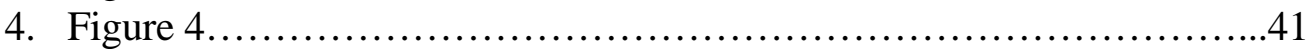

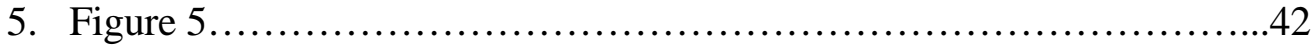

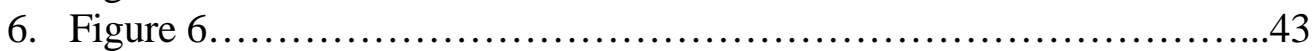

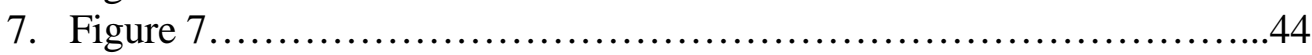

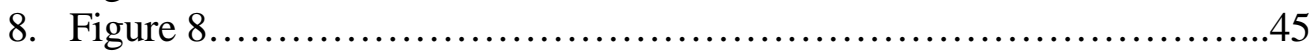

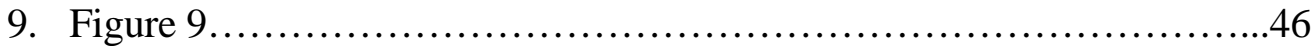

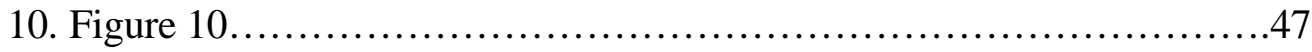

References.............................................................48

Curriculum Vitae....................................................50 


\section{LIST OF SYMBOLS / NOMENCLATURE}

1. AL - antennal lobe

2. BMI - bicuculline

3. $\mathrm{CR}$ - conditioned response

4. CS - conditioned stimulus

5. CS- - un-reinforced conditioned stimulus

6. $\mathrm{CS}+-$ reinforced conditioning stimulus

7. EMG - electromyography

8. GLM - general linear model

9. HEX - 2-hexanone

10. LNs - local interneurons

11. LOL - racemic linalool

12. MES - methyl salicylate

13. NER - cis-nerolidol

14. OB - olfactory bulb

15. OCT - 2-octanone

16. ORN - olfactory receptor neuron

17. PCT - picrotoxin

18. US - unconditioned stimulus

19. ZHP - cis-3-hexenyl proprionate 


\section{N T R O D U C T I O N}

Due to the highly dynamic spatial-temporal structure of olfactory plumes, odor cues are experienced by organisms in a highly variable manner. As an organism moves through an odor plume, the interaction of odorant molecules on the organism's sensory array produces a highly variable input signal (Vickers et al., 2001). Therefore, if organisms are to successfully exploit olfactory cues within this highly variable environment, their olfactory processing systems must detect and discriminate among a myriad of odorants and odor blends while maintaining some level of constancy across a reasonably broad range of intensities.

Studies have suggested that olfactory systems of both vertebrates and invertebrates detect odors based on the interaction of specific molecular features of odorant molecules with olfactory receptor neurons (ORNs) (Hildebrand, 1996; Galizia et al., 1999; Sachse, et al., 1999). At the first synaptic relay, the antennal lobe (AL) in insects and olfactory bulb (OB) in vertebrates, input from the sensory array produces a distinct spatial combination of olfactory glomeruli activation for each discriminable odorant (Shepherd 1991; Cinelli et al. 1995; Joerges et al., 1997). The uniqueness of this activation pattern is correlated to an animal's ability to discriminate among closely related odorants (Linster et al., 2001; Linster et al 2002).

It has also been shown that changing stimulus concentration can elicit substantive changes in spatial activation patterns in the glomeruli, including glomerular recruitment and loss (Galizia et al., 2000). These changes could equate to changes in the amount of information available to the animal. For instance, at lower concentrations, the animal may perceive the presence of an odorant stimulus but is unable to identify the odorant or discriminate it from other odorants. At higher concentrations, however, when spatial patterns involve a greater percentage of the AL, 
there is more information available to the animal from which to produce a more unique percept of the given odor stimulus. In an attempt to resolve these issues, our first experiment, which is outlined in chapter 1 , was designed to behaviorally define stimulus detection thresholds for different odors and investigate changes in responsiveness as a function of stimulus concentration. Using a Pavlovian olfactory-learning paradigm, we characterized the ability of the moth Manduca sexta to detect and respond to monomolecular odorants as a function of stimulus concentration. Manduca sexta is a favorable comparative model system because like the honeybee, this moth readily learns odor-food relationships in a Pavlovian olfactory conditioning paradigm (Daly and Smith, 2000; Daly et al., 2001a,b). In addition, this model system is readily amenable to a number of neurophysiological measures which allow detailed anatomical and statistical analysis of stimulus-dependent responses (Daly et al., 2004).

Further behavioral studies of several insects including several moth species have revealed that the olfactory systems of insects can readily discriminate among a wide variety of odorants (Balkenius and Kelber, 2006; Daly et al., 2001b; Fan and Hansson, 2001; Laska et al., 1999; Sakura et al., 2002; Skiri et al., 2005; Wright et al., 2005; Wright et al., 2002). These studies typically use stimulus generalization and differential conditioning protocols, which are important behavioral paradigms for investigating the perceptual relatedness of stimuli. Stimulus generalization can be defined as the degree to which a novel stimulus elicits the conditioned response, based on its overall perceptual similarity to the conditioning stimulus (CS) used in the prior formation of the CR (Daly et al., 2001b). Discrimination on the other hand is the ability to establish that two or more stimuli are different. Typically, discrimination experiments are based on the product of differential reinforcement of one stimulus (CS+), with non-reinforcement of a second (CS-; (Daly et al., 2001b; Daly and Smith, 2000). Both generalization and discrimination 
experiments have been used to investigate the discriminability of subtly different monomolecular odorants based on physical characteristics such as carbon chain length (Bhagavan and Smith, 1997; Cleland et al., 2002; Cleland and Narla, 2003; Daly et al., 2001a) thereby providing basic information about which stimulus dimensions are relevant to odor coding in olfactory systems. More over, behavior-pharmacological investigations have begun to unravel the complex relationship between AL function and olfactory acuity (Hosler et al., 2000; Stopfer et al., 1997). Our goal for the second experiment was to behaviorally characterize the effect of stimulus concentration on discrimination learning and performance using a discrimination learning assay (Daly and Smith, 2000; Daly et al., 2001b); this study is outlined in chapter 2.

It is also now known that the ability of animals to perceive and discriminate a seemingly limitless number of odors and odor blends is dependent on a relatively limited number of olfactory receptor types. Each receptor type is expressed individually within a given subset of olfactory receptor neurons, which converge into individual glomerular structures; this pattern is common in many holometabolous insects such as M. sexta and vertebrates (Hildebrand and Shepherd, 1997). This input is further mediated by an array of local interneurons, which are primarily inhibitory and can be classified into a number of morphologies in insects (Christensen et al., 1993; Matsumoto and Hildebrand, 1981) and vertebrates (Shipley and Ennis, 1996). Several neuromodulators and neurotransmitters play important roles in local information processing in both the vertebrate OB (Wang et al., 1986) and in the insect AL (Hildebrand and Shepherd, 1997; Shipley and Ennis, 1996). Of particular interest is the inhibitory neurotransmitter GABA ( $\gamma$-aminobutyric acid). In the AL, there are local interneurons (LNs) which are primarily GABAergic inhibitory neurons with broad multiglomerular ramifications 
(Leitch and Laurent, 1996; Matsumoto and Hildebrand, 1981) as well as other morphologies which have more restricted ramification patterns (Christensen et al., 1993). Thus, as with vertebrates, there appears to be several classes of LNs with potentially distinct functional roles. GABAergic transmission is involved in restricting spatial patterns of glomerular activation (Sachse and Galizia, 2002) as well as potentially generating temporal patterns of activity within the AL that may be critical for representing olfactory information in other central nervous system regions (Daly et al., 2004; Laurent and Davidowitz, 1994; Laurent et al., 2001). For instance, honeybee studies proposed that GABA regulates the specificity of associative olfactory memory formations (Hosler et al., 2000) by mediating the transient synchronization of distributed AL output neurons on an oscillatory timescale (Stopfer et al., 1997). Synchronization could be abolished in these studies by application of picrotoxin (PCT), an antagonist of the $\mathrm{GABA}_{\mathrm{A}}$ receptor, leading to impairment of discrimination of similar but not dissimilar odors. These results were obtained using a stimulus generalization paradigm. As mentioned above, stimulus generalization specifically asks whether odors are perceived as similar, it does not establish whether the animal can discriminate per se.

Thus to more carefully assess the effect of impairment of local inhibitory processing within the AL on the ability to resolve odor signals, we performed comparative studies in $M$. sexta as outlined in chapter 3. Here we implemented both stimulus generalization and differential conditioning paradigms. Furthermore, we used multiple $\mathrm{GABA}_{\mathrm{A}}$ antagonists: bicuculline (BMI), which is known to effectively block $\mathrm{GABA}_{\mathrm{A}}$ function in $M$. sexta as well as picrotoxin (PCT), which has been used in previous behavior-pharmacological studies (Hosler et al., 2000; Stopfer et al., 1997). We predicted that the differential conditioning paradigm would establish that $\mathrm{GABA}_{\mathrm{A}}$ blockade causes a more systemic disruption of olfactory acuity and not simply impair 
discrimination of molecularly similar odors as shown in the generalization paradigm. This prediction was based on the theoretical supposition that dissimilar odors may be so different perceptually that even though the animal has a greater difficulty distinguishing between them under conditions of GABA disruption, they may nevertheless continue to be perceptually "unlike" the conditioning odor (see Figure 1). To further characterize how systemic the disruption by $\mathrm{GABA}_{\mathrm{A}}$ blockade may be, we also quantified its effects on detection thresholds. In this case, if detection thresholds were impaired by $\mathrm{GABA}_{\mathrm{A}}$ blockade, then it stands to reason that changes in detection are the underlying cause of the loss of ability to discriminate. 


\section{CHAPTER 1: Psychophysical measuresofodor de tection ${ }^{1}$}

\section{Introduction}

Our first experiment was designed to behaviorally define stimulus detection thresholds for different odors and investigate changes in responsiveness as a function of stimulus concentration. A Pavlovian-based olfactory conditioning paradigm was used to condition moths using neat stimulus concentrations followed by testing across a dilution series. The primary goal was to establish the lowest concentration at which moths first start to respond to odors with a conditioned response; this is our behaviorally defined detection threshold. Previous studies of olfactory learning and memory in the honeybee demonstrated that as the concentration of a stimulus was lowered, its salience (i.e. its perceived intensity) was lowered (Pelz, Gerber, and Menzel, 1997; Skiri, Stranden, Sandoz, Menzel, and Mustaparta, 2005; Wright and Smith, 2004). The perceptual salience of a given stimulus is a key factor that directly influences the rate of learning (Rescorla and Wagner, 1972). Hence, as concentration is lowered, assuming all else equal, salience is lowered and learning is hampered; this in turn will bias threshold measures to be higher. As an alternative approach, we conditioned animals at high stimulus concentrations and tested with a panel of increasing concentration. This ensures that the overall learning is roughly comparable across groups. This approach assumes that at the level of odor perception, salience changes but identity does not; our prior research with the odors used herein confirms this assumption is not violated (Daly et al., in press).

\footnotetext{
${ }^{1}$ This preliminary work was done in collaboration with Lynnsey A. Carrell as part of her Honor's thesis and has been submitted and accepted for publication; (Daly et al., in press).
} 


\section{Materials and Methods}

\section{Subjects}

Male and female $M$. sexta were obtained at or near stage 18 of pupal development from Arizona Research Labs, Division of Neurobiology via overnight delivery. Upon arrival, pupae were isolated in brown paper bags where they remained undisturbed until used. Bags were placed in an environmental control chamber that held temperature at $25^{\circ} \mathrm{C}, 90 \%$ relative humidity and a reverse 16/8 L/D cycle. Eclosion dates were recorded once daily on bags in which newly emerged adults were found. Age at initiation of training was between 5-7 days post eclosion to increase motivation to feed without hindering performance (Daly and Smith, 2000). Experiments were run during the dark period of the cycle. Subjects were randomly assigned in approximately equal numbers of males and females to one experimental group and used only once.

\section{Preparation:}

Moths were placed in a $1 / 2$ in ID tube and restrained with pipe cleaner and tape. The proboscis was extended and threaded into a $4 \mathrm{~cm}$ length of Tygon brand surgical tubing. This tubing was then attached to the tube containing the moth with a soft wax. An electrode was then placed into the right side of the head capsule such that the electrode made contact with the pharyngeal dilator muscle (Eaton, 1971). This is one of many feeding related muscles that can be observed using electromyography (EMG) and provides a meaningful indication of feeding responses to the conditioning odor (CS), test odors, as well as to the unconditioned stimulus (US). A reference electrode was also placed in the contralateral eye. During conditioning and testing, the plastic tube containing the prepared moth was "plugged" into a stand which held the moth upright and the electrodes were connected to a WPI brand DAM 50 amplifier. This allowed for the rapid 
sequential swapping of many animals onto the conditioning and testing stage using only a single differential amplifier. Finally, the amplified EMG signal was fed to a speaker and an oscilloscope to provide both auditory and visual indicators of feeding behavior (Daly and Smith, 2000; Daly et al., 2001a,b).

\section{Stimulus Delivery:}

The conditioning stage consisted of an odor delivery system and an odor evacuation vent. Animals were placed into the threshold of the evacuation vent where a steady stream of air blew by the animals at a rate of $0.2-0.3 \mathrm{~m} / \mathrm{s}$. Air flow was measured by a Fisher brand hotwire anemometer. An odor cartridge was placed approximately $10 \mathrm{~cm}$ upwind and aimed directly at the moth's head. Distance from the cartridge to the moth ensured adequate dispersion over the entire antennae; this has been confirmed with titanium-tetrachloride (liquid smoke) tests (Daly and Smith, 2000; Daly et al., 2001a,b). Airflow through the odorant cartridge, as well as CS/US timing was controlled by a programmable logic chip (PLC). Filtered air was supplied via a central air line. Air was first passed through a 500 cc Drierite brand cartridge to extract moisture then passed through a 500 cc active charcoal filter. Output from the filter array then passed through a flow meter, which was set at $250 \mathrm{ml} / \mathrm{min}$, and into a Lee brand 3-way valve, which was controlled by the PLC. Air blew into one port on the valve then immediately out a second port. When the valve was activated, the output was shunted to the third port which was connected via Tygon brand tubing to the odorant cartridge. Odorant cartridges were fashioned from glass tubing (6 mm ID) cut to a length of $7 \mathrm{~cm}$. Cole-Parmer brand nylon lure-fittings were inserted into either end of the glass tube. The internal volume of the cartridge was $\sim 1.5 \mathrm{ml}$ after the fittings were inserted. Given this cartridge size, a flow rate of $250 \mathrm{~ms} / \mathrm{min}$ and assuming no 
mixing, it should take an estimated 0.36 seconds for the air volume of the cartridge to be replaced (Daly and Smith, 2000; Daly et al., 2001a,b).

Six odorants were used in the current study: linalool (LOL), cis-nerolidol (NER), cis-3hexenyl proprionate (ZHP), methyl salicylate (MES), 2-hexanone (HEX), and 2-octanone (OCT). The first 4 were selected based on prior evidence suggesting that these are possibly female-specific host-plant volatiles (Shields and Hildebrand, 2001; Fraser 2003). The final two odorants were selected based on their successful use in prior olfactory learning experiments (Daly and Smith, 2000; Daly et al., 2001a,b). All odorants were 97\% pure or better. Inserted into the cartridge was a piece of Whitman brand No. 3 white filter paper. Odorants were placed onto the filter paper prior to use. A five log step range of concentrations was established (0.5ng/ $\mathrm{l}$, $5 \mathrm{ng} / \mu \mathrm{l}, 50 \mathrm{ng} / \mu \mathrm{l}, 0.5 \mu \mathrm{g} / \mu \mathrm{l}$ and $5 \mu \mathrm{g} / \mu \mathrm{l})$; concentrations were based on dilution in mineral oil. When testing with these concentrations, a $2 \mu \mathrm{l}$ aliquot was placed on a small strip of filter paper inside a glass cartridge. This produced a final concentration range from $10 \mathrm{ng} / \mu \mathrm{l}$ to $10 \mu \mathrm{g} / \mu \mathrm{l}$. When conditioning, a $3 \mu \mathrm{l}$ aliquot of neat odorant was used. The intended goal of using neat odorant was to ensure that moths were experiencing the most salient stimulus possible. This should ensure that a lack of a conditioned response during testing could not be attributed to a lack of learning due to lower salience (Rescolra and Wagner, 1972; Wright and Smith, 2004).

\section{Conditioning and testing protocols:}

To establish behavioral measures of detection thresholds, a forward-paired Pavlovian-based olfactory conditioning protocol was used (Daly and Smith, 2000). Moths were conditioned and tested in one of six groups ( $\mathrm{N}=60$ /group) and experienced only one of the 6 odorants. Each moth was presented with a 4s CS using neat odorant. Three seconds into CS delivery, the US, a $5 \mu \mathrm{l}$ droplet of $0.75 \mathrm{M}$ sucrose solution, was applied to the partially extended proboscis via a Gilmont 
brand syringe. US delivery was also 4s in duration. This basic CS-US pairing was repeated 6 times in 6 min intervals. Following conditioning, moths were returned to the environmental control chamber until testing at 24h and 48h post conditioning. Since high concentrations were used for conditioning, this minimum 24h post conditioning wait eliminated any effects of sensory adaptation/fatigue.

Behavioral response measures used were based on changes in the rate of EMG activity from the cibarial pump muscle (Daly and Smith, 2000). Subjects were scored based on a detected increase in feeding behavior upon presentation of the odor. During conditioning trials, any increased feeding activity prior to presentation of sucrose was recorded as a conditioned response (CR) for that trial; this was used to index acquisition of the CR. During test trials, a 4s period spanning the total time of odor presentation was used to score behavioral responses. In general, data were recorded as 0 for no response and 1 for a CR. Moths were first presented with a blank stimulus to assess baseline responsiveness to non olfactory cues that might be associated with the puffing of air; this provided a comparative control for all non-olfactory responses. They were then presented with test odorant stimuli at the concentrations described above. Test stimuli were always delivered sequentially from lowest to highest in order to avoid extinction effects (Daly and Smith, 2000). That is, un-reinforced presentation of higher concentrations prior to presentation of lower concentrations could result in extinction effects at lower concentrations on subsequent trials; this would bias results towards higher thresholds.

\section{Data Analysis}

General linear modeling (GLM) was used to analyze variation in CR probability as a function of the moths' sex (M, F), testing day (24h, 48h), odor (HEX, LOL, MES, NER, OCT \& ZHP) and concentration $(0,0.001,0.01,0.1,1, \& 10 \mu \mathrm{g} / \mu \mathrm{l})$. These variables were treated as categorical 
variables. GLM analysis was used because it allows for theoretical pre-specification of variables and hierarchically partitions variance components for both categorical and continuous variables (Cohen and Cohen, 1983).

All possible two and three way interactions were tested with significance threshold set at ( $\mathrm{p}<$ 0.01) to reduce experiment-wise error rate. Tukey's HSD post hoc analysis was performed in SAS $(\mathrm{p}<0.05)$. Curvilinear regression functions were calculated in Excel to predict population mean CR probability as a function of concentration; for this analysis asymptotic functions created using a third order polynomial function of CR probability were used to calculate each regression line. Third order polynomials produced the best general fits for all odorants.

\section{Results}

The overall statistical model explaining variation in CR-probability for this experiment was significant $(\mathrm{p}<0.0001)$. Results indicate a main effect of odor $(\mathrm{p}<0.01)$. As shown in Figure 2a, Tukey's post hoc analysis indicated that moths were differentially more responsive to ZHP and less responsive to MES and NER relative to HEX, LOL and OCT. The main effect of concentration was also significant $(\mathrm{p}<0.01)$. Tukey's post hoc analysis of this variable indicated that increasing concentrations produced increasing CR probabilities. Figure 2b shows a general stepwise increase in CR probability as a curvilinear function of increasing concentration.

In addition, there was a significant two-way interaction of odor-by-concentration $(\mathrm{p}<0.01)$. This indicates that the rate of increase in CR probability as a function of concentration was dependent on the odorant used. This interaction resulted in different odors producing different concentration response functions as modeled by third order polynomials shown in Figure 3.

Furthermore, post hoc comparisons of the zero-odor control with each stepwise increase in concentration (1-tailed paired t-test; $\mathrm{p}<0.05$ ) indicated that detection was odor-dependent 
(identified by $\left(^{*}\right)$ inset for each odorant in Figure 3). Results of this analysis indicate that moths were most sensitive to LOL, as indicated by a significant responsiveness at $0.001 \mu \mathrm{g} / \mu \mathrm{l}$, followed by HEX, OCT and ZHP $(0.01 / \mu \mathrm{l} \mu \mathrm{g})$, MES $(0.1 / \mu \mathrm{l} \mu \mathrm{g})$ and NER $(10 \mu \mathrm{g} / \mu \mathrm{l})$.

\section{Discussion}

The overall goal of this study was to psychophysically quantify odor detection thresholds and characterize the change in these measures as a function of increasing stimulus concentration. Our results indicated that odor detection thresholds varied widely with different odors. These results were also compared to electroantennogram (EAG; Daly et al., in press). Results from this analysis clearly indicated a very high correspondence between behavioral and neurophysiological responses across the presented odorant and its concentration (Daly et al., in press). In fact, these correlations remained high across more complex interactions between factors. These results strongly support the conclusion that the behavioral assay used herein provides an accurate psychophysical measure of sensory acuity. Therefore, we applied the same assay in subsequent experiments to establish discrimination thresholds as well as assess the effects of $\mathrm{GABA}_{\mathrm{A}}$ blockade on olfactory acuity. 


\section{$\begin{array}{llllll}\text { CHA P TER } & \text { 2: The effect } & \text { Th } & \text { intensity }\end{array}$} discrimination learning and performance

\section{Introduction}

Our goal for the second experiment was to behaviorally characterize the effect of stimulus concentration on discrimination learning and performance using a discrimination learning assay (Daly and Smith, 2000; Daly et al., 2001b). Discrimination learning experiments have been used, for example, to investigate the discriminability of subtly different monomolecular odorants based on physical characteristics such as carbon chain length (Smith, 1993). Previous studies of discrimination-learning as a function of concentration have recognized that as the concentration of a stimulus is lowered, its salience is lowered as well (Pelz et al., 1997; Wright and Smith, 2004). Stimulus salience is a key factor that directly influences the rate of learning (Rescorla and Wagner, 1972). Hence, as salience is lowered learning is hampered; this in turn positively biases discrimination threshold measures. Typically, this is compensated for by adding more learning trials. However, given that insects such as honeybees and moths rapidly change in their motivational states, conditioning to a criterion becomes problematic. An alternative approach that we apply herein is to simply condition animals at high stimulus concentrations and test with a panel of increasing concentration. This ensures that the overall learning is roughly comparable across groups. This approach assumes that at the level of odor perception, salience changes but identity does not; our prior research with the odors used herein confirms this assumption is not violated (Daly et al., in press).

\footnotetext{
${ }^{2}$ This work was done in collaboration with Lynnsey A. Carrell and has been submitted; (Daly et al., in review).
} 


\section{Materials and Methods}

\section{Subjects}

Male and female $M$. sexta were obtained at or near stage 18 of pupal development from Arizona Research Labs, Division of Neurobiology via overnight delivery. Upon arrival, pupae were isolated in brown paper bags where they remained undisturbed until used. Bags were placed in an environmental control chamber that held temperature at $25^{\circ} \mathrm{C}, 90 \%$ relative humidity and a reverse 16/8 L/D cycle. Eclosion dates were recorded once daily on bags in which newly emerged adults were found. Age at initiation of training was between 5-7 days post eclosion to increase motivation to feed without hindering performance (Daly and Smith, 2000). Experiments were run during the dark period of the cycle. Subjects were randomly assigned in approximately equal numbers of males and females to one experimental group and used only once.

\section{Preparation:}

Moths were placed in a $1 / 2$ in ID tube and restrained with pipe cleaner and tape. The proboscis was extended and threaded into a $4 \mathrm{~cm}$ length of Tygon brand surgical tubing. This tubing was then attached to the tube containing the moth with a soft wax. An electrode was then placed into the right side of the head capsule such that the electrode made contact with the pharyngeal dilator muscle (Eaton, 1971). This is one of many feeding related muscles that can be observed using electromyography (EMG) and provides a meaningful indication of feeding responses to the conditioning odor (CS), test odors, as well as to the unconditioned stimulus (US). A reference electrode was also placed in the contralateral eye. During conditioning and testing, the plastic tube containing the prepared moth was "plugged" onto a stand which held the moth upright and was connected to a WPI brand DAM 50 amplifier. This allowed for the rapid sequential swapping of many animals onto the conditioning and testing stage using only a single channel amplifier. 
Finally, the amplified EMG signal was fed to a speaker and an oscilloscope to provide both auditory and visual indicators of feeding behavior (Daly and Smith, 2000; Daly et al., 2001a,b).

\section{Stimulus Delivery:}

The conditioning stage consisted of an odor delivery system and an odor evacuation vent. Animals were placed into the threshold of the evacuation vent where a steady stream of air flew by the animals at a rate of 0.2-0.3 m/s. Air flow was measured by a Fisher brand hotwire anemometer. An odor cartridge was placed approximately $10 \mathrm{~cm}$ upwind and aimed directly at the moth's head. Distance from the cartridge to the moth ensured adequate dispersion over the entire antennae; this has been confirmed with titanium-tetrachloride (liquid smoke) tests (Daly and Smith, 2000; Daly et al., 2001a,b). Airflow through the odorant cartridge, as well as CS/US timing was controlled by a programmable logic chip (PLC). Filtered air was supplied via a central air line. Air was first passed through a 500 cc Drierite brand cartridge to extract moisture then passed through a 500 cc active charcoal filter. Output from the filter array then passed through a flow meter, which was set at 250 $\mathrm{ml} / \mathrm{min}$, and into a Lee brand 3-way valve, which was controlled by the PLC. Air flew into one port on the valve then immediately out a second port. When the valve was activated, the output was shunted to the third port which was connected via Tygon brand tubing to the odorant cartridge. Odorant cartridges were fashioned from glass tubing (6 mm ID) cut to a length of $7 \mathrm{~cm}$. ColeParmer brand nylon lure-fittings were inserted into either end of the glass tube. The internal volume of the cartridge was $\sim 1.5 \mathrm{ml}$ after the fittings were inserted. Given this cartridge size, a flow rate of $250 \mathrm{~ms} / \mathrm{min}$ and assuming no mixing, it should take an estimated 0.36 seconds for the air volume of the cartridge to be replaced (Daly and Smith, 2000; Daly et al., 2001a,b). 


\section{Conditioning and testing protocols:}

To establish discrimination thresholds, a discrimination learning protocol was used where one odorant $(\mathrm{CS}+)$ was forward-paired with the US as described in experiment 1. Pseudo randomly interspersed between forward paired conditioning trials were trials where a second odorant (CS-) was presented for $4 \mathrm{~s}$ but was not paired with the US. Two pseudo randomized sequences were used on different subgroups of moths to ensure the CS+ was preceded and followed equally by the CS-. A total of 4 groups ( $\mathrm{N}=120$ moths each) were differentially conditioned with LOL and one of following four odorants: NER, MES, ZHP or HEX. An additional group $(\mathrm{N}=120)$ was differentially conditioned with HEX and OCT. Selection of comparisons was based on results of Experiment 1 with the aim of characterizing discrimination of odors with a range of detection thresholds. Groups were counterbalanced so that half of each group of moths ( $\mathrm{N}=60$ ea) were conditioned with one odor as the CS+ and the other as the CS-; for the second half of the group, the CS+ and CS- odors were reversed. This allowed us to determine whether discrimination was symmetric between reinforced odors as a function of differences in detection thresholds. Moths were tested as described in experiment 1 but with both CS+ and CS- in a pseudo random manner.

\section{Data Analysis}

A number of variables were created to explain the variation in CR probability. The key variable for this experiment was concentration, which was treated as a continuous variable. An additional key variable was the conditioning stimulus, a categorical variable that indicated whether an odorant was used as the CS+ or the CS-. The variable odor, on the other hand, was a categorical variable that specified the identity of the test odorant. The variable treatment indicated which pair wise combination of odorants was used for a given group and was treated as a categorical variable. 
GLM analysis was used to analyze variation in CR probability as a function of the variables described above. Variables were always hierarchically entered into the GLM so that the effects of CS and concentration were extracted after all other main effects and associated interactions were accounted for first. All possible interactions were tested and significance threshold was set at $(\mathrm{p}<$ 0.01) to reduce experiment-wise error rate. A post hoc analysis using 1-tailed paired t-tests was performed in SAS. Asymptotic curvilinear regression functions (created using a third order polynomial) were calculated to predict population mean CR probability as a function of concentration.

\section{Results}

The overall statistical model explaining variation in CR-probability for this experiment was significant $(\mathrm{p}<0.0001)$.There were three variables that were of primary interest to this study and they all produced significant effects across all odor pairs. These were CS, concentration and their interaction; all of these variables were significant $(\mathrm{p}<0.01)$. As expected, the significant effect of CS indicated that the CS+ and the CS- conditions produced different response probabilities. The significant interaction of CS and concentration indicated that there was a systematic divergence in CR probability as a function of increasing concentration; this effect is evident in Figure 4.

Figure 4 also displays the mean CR probability by concentration the for each odor pair. These are further subdivided so that each odor is displayed as both the CS+ and CS-. For each CS+/CSpair, one-tailed pair wise t-tests were used to compare the CR probability for the CS+ and CS- at increasing steps in concentration until the concentration at which there was a significant difference between the two. Note that for all odor pairs, except HEX/OCT, different discrimination thresholds were identified across the counterbalance. For example, in all cases where LOL was the CS+ in a pair, the discrimination threshold, as identified therein was 1 or more orders of magnitude lower 
than when LOL was the CS- for a given odor pair . In one most extreme case (NER/LOL), when NER was the CS+, no significant evidence of discrimination was observed. This lack of divergence can be accounted for by the main effect of odor whereby NER generally elicits a low CR probability. Indeed this case is comparable to NER response in experiment 1 (see Fig. 3f and 4b).

\section{Discussion}

The problem of identifying discrimination thresholds and psychophysical measures of differential concentration-response functions is daunting in invertebrates because of the limited behavioral paradigms at our disposal. Nevertheless, we have shown in chapter 1 that detection thresholds and concentration-response functions can be generated using a Pavlovian paradigm and that this general method correlates well with matched physiological measures (Daly et al., in press). In the current experiment, we provide differential concentration-response functions for pairs of odors. By comparing this experiment with results observed in Chapter 1, we conclude that discrimination must be a process that is unique though dependent on detection. We make this conclusion based on the observation that moths detect these odors at concentrations 1 or more orders of magnitude below what is necessary for discrimination (Daly at al., in review).

Nevertheless, it is necessary to note that defining discrimination thresholds is more complex than simply assessing the concentration at which animals produce a significant differential CR to the reinforced and non reinforced odors because equal dilutions, based on the density of the individual odors, do not equate to equivalent saliences at the level of sensory perception. This is because density does not strictly equate to volatility. Furthermore, accounting for the physical properties of odorants is insufficient given that the antennal and sensory system is clearly differentially sensitive to different odors. Nevertheless, when we compare asymmetries in the detection thresholds observed in chapter 1 with the asymmetries in discrimination thresholds, it 
becomes clear that in all cases, the asymmetries in differential concentration-response functions are predicted by differences in detection thresholds. For example, LOL is detectable at $0.001 \mu \mathrm{g} / \mu \mathrm{l}$ while MES is detectable at $0.1 \mu \mathrm{g} / \mu \mathrm{l}$. When LOL is the reinforced odor, it's discrimination from MES occurs at $0.01 \mu \mathrm{g} / \mu \mathrm{l}$ while when MES is reinforced, it's discriminated from LOL occurs at $1.0 \mu \mathrm{g} / \mu \mathrm{l}$. Therefore, discrimination thresholds must be defined in these cases based on which odor of the pair has the higher detection threshold. In this case, we satisfy the requirement that both odors can be detected. 


\section{CHAPTER 3: The effect of GABA blockade on psychophysical measures of odor detection and discrimination ${ }^{3}$}

\section{Introduction}

The inhibitory neurotransmitter GABA, is important in local information processing in both the olfactory bulb (OB; Wang et al., 2001) and antennal lobe (AL; Hildebrand and Shepherd, 1997; Shipley and Ennis, 1996). In the AL, there are local interneurons (LNs) which are primarily GABAergic inhibitory neurons with broad multiglomerular ramifications (Leitch and Laurent, 1996; Matsumoto and Hildebrand, 1981) as well as other morphologies which have more restricted ramification patterns (Christensen et al., 1993). Thus, as with vertebrates, there appears to be several classes of LNs with potentially distinct functional roles. GABAergic transmission is involved in restricting spatial patterns of glomerular activation (Sachse and Galizia, 2002) as well as potentially generating temporal patterns of activity within the AL that may be critical for representing olfactory information in other central nervous system regions (Daly et al., 2004; Laurent and Davidowitz, 1994; Laurent et al., 2001).

Associated with these changes in AL function, studies of olfactory discrimination in honey bees have suggested that blockade of GABAergic transmission disrupts discrimination of similar but not dissimilar odorants (Stopfer et al., 1997). These results were obtained using a stimulus generalization paradigm, which specifically asks whether odors are perceived as similar; it does not establish whether the animal can discriminate per se. Our third experiment was aimed at assessing the effect of GABA blockade within the AL of M. sexta on the ability to resolve odor signals; we 
performed comparative studies using both stimulus generalization and differential conditioning paradigms. In addition, we used multiple $\mathrm{GABA}_{\mathrm{A}}$ antagonists: $\mathrm{BMI}$, which is known to effectively block $\mathrm{GABA}_{\mathrm{A}}$ function in $M$. sexta and PCT, which has been used in previous behaviorpharmacological studies (Hosler et al., 2000; Stopfer et al., 1997). We predicted that the differential conditioning paradigm would establish that $\mathrm{GABA}_{\mathrm{A}}$ blockade causes a more systemic disruption of olfactory acuity and not simply impair discrimination of molecularly similar odors as shown in the generalization paradigm. This prediction was based on the theoretical supposition that dissimilar odors may be so different perceptually that even though the animal has a greater difficulty distinguishing between them under conditions of GABA disruption, they may nevertheless continue to be perceptually "unlike" the conditioning odor (see Figure 1).

To further characterize how systemic the disruption by $\mathrm{GABA}_{\mathrm{A}}$ blockade may be, we also quantified its effects on detection thresholds. In this case, if detection thresholds were impaired by $\mathrm{GABA}_{\mathrm{A}}$ blockade, then it stands to reason that changes in detection are the underlying cause of the loss of ability to discriminate.

\footnotetext{
${ }^{3}$ This work has been submitted; (Mwilaria and Daly, in review).
} 


\section{Materials and Methods}

\section{Subjects}

Male and female Manduca sexta were obtained at or near stage 18 of pupal development from Arizona Research Labs, Division of Neurobiology via overnight delivery. Upon arrival, pupae were isolated in brown paper bags where they remained undisturbed until used. Bags were placed in an incubator that holds temperature at $25^{\circ} \mathrm{C}, 90 \%$ relative humidity and a reverse $16 / 8$ L/D cycle. Eclosion dates were recorded once daily on bags in which newly emerged adults were found. Age at initiation of conditioning was between 5-7 days post eclosion to increase motivation to feed (Daly and Smith, 2000). Experiments were performed during the dark period of the L/D cycle. Subjects were randomly assigned in approximately equal numbers of males and females to one experimental group and used only once.

\section{Preparation}

Moths were inserted head first into a snugly-fitting plastic tube with the head protruding out and over a tab at the end of the tube. The exposed back of the body up to the head was then firmly shackled to the tab with a piece of tape. This method immobilized the insect and provided a secure platform for the head in preparation for dissection. After removing all scales from the head capsule, a single-ended EMG electrode was placed through the cuticle just above the left cibarial pump muscle, and a reference electrode was placed in the contra lateral eye. Electrode impedance was tested using an FHC low voltage impendence meter to confirm electrode circuit quality with the cibarial pump muscle, a large muscle involved in feeding (Eaton, 1971). The proboscis was threaded through a $5 \mathrm{~cm}(0.5 \mathrm{~mm}$ ID) length of Tygon tubing and affixed to the plastic tube with a piece of soft wax. At this point, the moth was ready for the conditioning phase of the experiment. 


\section{Surgery and Injection Procedure}

Prior to testing, the caudal end of the head capsule of each conditioned moth was opened, thus exposing both ALs without having to remove the proboscis and associated musculature. However, it was necessary to reposition the pharyngeal dilator muscle forward for clearer access to the AL. The patch of cuticle with the muscle attached was simply sectioned then slid forward into the previously opened area and re-adhered to the head capsule with super glue. This procedure has been successfully used in previous studies and has no overt effect on the animal's ability to elicit normal feeding behavior (Daly et al., 2004).

The injection procedures are an enhancement of the methods described by Stopfer et al., (1997) and Hosler et al., (2000). As opposed to spritzing topically upon the exposed AL, a sharp quartz intracellular electrode was used to produce a wispy slow-tapering injection probe that was used to pierce and directly inject into the approximate center of the AL. The tips of these wispy intracellular probes were sheared using fine forceps to produce a relatively larger $10 \mu \mathrm{m}$ diameter opening.

The use of relatively sturdier quartz glass made it possible to pierce the protein sheath surrounding the AL without removing it. The narrow tip minimized damage to the AL while the slow-tapering shaft provided consistent calibration from injection even when minor chipping of the tip occurred. Injections were controlled by a General Valve brand Picospritzer II. Each probe was calibrated to produce a standard droplet volume estimated at $\sim 2 \mathrm{nl}$, using consistent injection pressure of 20 psi and varying injection time. This estimate was calculated by injecting into a mineral oil pool and measuring the diameter of the droplet sphere under a dissecting scope. Additionally, after the injection of each animal, the probe was again tested in this manner to confirm calibration. If the probe was found to be clogged or out of calibration, that animal was 
disqualified from the study. It should be noted that this method differs from Waldrop et al., (1987) and Christensen et al., (1998) who superfused at 13 psi for up to 5 mins in desheathed and isolated brains. They also used a $\sim 60 \mu \mathrm{m}$ diameter multibarrel pipette with individual barrel inner diameters of approximately $20 \mu \mathrm{m}$. Thus the method implemented here should be less intrusive by producing a smaller entrance, leaving the protective sheath intact and delivery of a far smaller bolus but at the cost of allowing injection of only one agent.

Control moths were injected with physiological saline solution containing (in mM): 150 $\mathrm{NaCl}, 3 \mathrm{CaCl}_{2}, 3 \mathrm{KCl}, 10 \mathrm{~N}$-tris[hydroxymethyl]methyl-2-aminoethanesulfonic acid buffer, and 25 sucrose, PH 6.9 (Christensen et al., 1993). Treatment groups were injected with either $100 \mu \mathrm{M}$ PCT or $2000 \mu \mathrm{M}$ BMI diluted in physiological saline. This PCT concentration was chosen because it has been shown to abolish inhibitory GABA-ergic feedback in locusts as well as honey bees (Stopfer et al., 1997). On the other hand, the BMI concentration used was one order of magnitude above the concentration used in previous $M$. sexta studies (Christensen et al., 1998). Here we injected a far smaller volume at higher concentration in an effort to optimize the effect of BMI while minimizing the injection effects.

\section{Stimulus Delivery:}

The conditioning stage consisted of an odor delivery system and an odor evacuation vent. Naïve moths were placed into the threshold of the evacuation vent where a steady stream of air flowed by the animals at a rate of $0.2-0.3 \mathrm{~m} / \mathrm{s}$. Air flow was measured by a Fisher hotwire anemometer. An odor cartridge was placed $10 \mathrm{~cm}$ upwind and aimed directly at the moth's head. Distance from the cartridge to the moth ensured adequate dispersion over both antennae; this has been confirmed with titanium-tetrachloride (liquid smoke) tests. Airflow through the odorant 
cartridge, as well as CS/US timing was controlled by a programmable logic chip (PLC). Filtered air was supplied via a central air line. Air was first passed through a 500 cc Drierite brand cartridge to extract moisture then passed through a 500 cc active charcoal filter. Output from the filter array then passed through a flow meter, which was set at $250 \mathrm{ml} / \mathrm{min}$, and into a Lee brand 3-way valve, which was controlled by the PLC. The final velocity of effluent from the nozzle was measured at $\sim 4$ ms via hotwire anemometer. Air flowed into the input port on the valve then immediately out a second, normally open exit port. When the valve was activated, the output was shunted to the third, normally closed exit port, which was connected via Tygon brand tubing to the odorant cartridge. Though difficult to measure, liquid smoke tests suggested that the flow from the nozzle decelerated to approximate the exhaust flow as it passed by the moth; hotwire anemometry did not indicate a measurable difference in flow at the position of the moth while the valve was activated.

Odorant cartridges were fashioned from glass tubing (6 mm ID) cut to a length of $7 \mathrm{~cm}$. Cole-Parmer brand nylon lure-fittings were inserted into either end of the glass tube. The internal volume of the cartridge was $1.5 \mathrm{ml}$ after the fittings were inserted. Given this cartridge size and a flow rate of $250 \mathrm{~ms} / \mathrm{min}$, it should take an estimated 0.36 seconds for the initial air volume of the cartridge to be replaced assuming no mixing.

Four odorants were used in the current study: LOL, MES, HEX, and OCT. The odorants were picked so that we had pairs of molecularly closely related and different odors (Stopfer et al., 1997). Detection thresholds for these odors were characterized in chapter 1(Daly et al., in press). All odorants were $97 \%$ pure or better. A five log step range of concentrations was established $(0.0005 \mu \mathrm{g} / \mu \mathrm{l}, 0.005 \mu \mathrm{g} / \mu \mathrm{l}, 0.05 \mu \mathrm{g} / \mu \mathrm{l}, 0.5 \mu \mathrm{g} / \mu \mathrm{l}$ and $5 \mu \mathrm{g} / \mu \mathrm{l})$ based on dilution in mineral oil. For conditioning, a $\sim 3 \mu$ laliquot of odor was placed on a strip of Whitman brand No. 3 white filter paper, which was then placed inside the glass cartridge. During testing, $2 \mu$ l aliquot was used. 


\section{Conditioning and Testing Protocols}

Experiment 3.1: The effect of $G A B A_{A}$ blockade on the generalization of a conditioned response.

Experimental and control groups were conditioned $24 \mathrm{~h}$ prior to testing. Conditioning was based on standard protocols (Daly and Smith, 2000). Briefly, during the conditioning phase, each animal received 6 forward pairings of the neat conditioning odor (CS) and sucrose solution (US; $5 \mathrm{~mL}$ of $0.75 \mathrm{M})$. For this experiment, OCT was used as the CS. There was a 6 min wait between conditioning trials. During each conditioning trial, a 4s puff of the CS was blown over the antennae followed by a 4s presentation of the US upon the proboscis. The timing of CS and US presentation was overlapped by 1 s.

After conditioning, animals were placed back into the environmental control chambers for 24h. Prior to testing, the ALs were surgically exposed and injected with either saline or a combination of saline and drug, held for $~ 10$ mins, and tested with neat concentrations of the CS (OCT), a similar odor (S; HEX), and dissimilar odor (D; MES). Odors were presented in random sequences; this general method is consistent with prior published reports (Hosler et al., 2000; Stopfer et al., 1997).

Two groups of 120 moths were used for each drug group; half were injected with saline and the other half with drug (either BMI or PCT). Injection and testing were performed by two researchers; one performed the injections and the other performed testing. The tester was blind to the treatment moths received. 


\section{Experiment 3.2: The effect of $G A B A_{A}$ blockade on discrimination thresholds}

Experimental and control groups were conditioned $24 \mathrm{~h}$ prior to testing. Conditioning was based on standard differential conditioning protocols (Daly and Smith, 2000). Briefly, during the conditioning phase, each animal received 6 forward pairings of the conditioning odor (CS+) followed by sucrose, and 6 un-reinforced trials of a second odor (CS-). CS+ and CS- were counterbalanced and presented in a pseudo random manner. During CS+ presentation, the CS and US were presented as previously described. The CS- was also presented in $4 \mathrm{~s}$ trials. After conditioning, animals were left for $24 \mathrm{~h}$ in the environmental control chambers. The ALs were then surgically exposed and injected with either saline or a combination of saline and drug, held for $\sim 10$ mins and tested with both CS+ and CS- odors at different concentrations in a log-step manner. Again, the tester was blind to the injection treatment. In each group, 240 moths were used whereby 120 were used with CS+/CS- and 120 with its counterbalance. Sixty of each subgroup of 120 moths were injected with saline and the other 60 with either BMI or PCT. The odor pairs used were OCT/HEX (similar odors) and LOL/MES (dissimilar odors).

\section{Experiment 3.3: The effect of $G A B A_{A}$ blockade on detection thresholds}

For this experiment, forward paired conditioning was performed as described in Experiment 3.1 and moths were again left for $24 \mathrm{~h}$ in the environmental control chambers prior to injection and testing. The same injection and testing protocols were used. Whereas in Experiment 3.1 moths were tested with the CS, S and D odors in a randomized manner, here moths were tested with a log step increase in concentrations of the CS beginning with blank and sequentially increasing the concentration. A total of 480 moths were used in this experiment; 120 moths were used for each of four odors used as a CS. As before, 60 of each group were injected with saline while the other 60 
were injected with BMI or PCT. All four odors (OCT, HEX, LOL, MES) were used and as before, the tester was always blind to the treatment given.

\section{Post-test assessment of feeding response}

Finally, to ensure that the effects of PCT and BMI were not attributable to a lack of ability to elicit the feeding response, moths were presented with sucrose upon the proboscis immediately after odor testing. Feeding responses were recorded in both saline and drug groups.

\section{Response Measures}

Behavioral response measures used have been established and detailed elsewhere (Daly and Smith, 2000). Measures were based on changes in the rate of EMG activity and/or extension of the proboscis (Daly and Smith, 2000). Subjects were scored based on a detected increase in feeding behavior upon presentation of the odor. During conditioning trials, any increased feeding activity during CS presentation but prior to US presentation was recorded as a CR for that trial; this was used to index acquisition of the conditioned response. During test trials, a 7s period was used to score behavioral responses; these data were used to assess the effects of treatment on generalization, discrimination and detection thresholds. In the final control experiment, any EMG activity and/or proboscis extension during sucrose application was recorded as a response.

\section{Analysis}

In Experiment 3.1, the primary interest was to quantify the relative change in CR-probability from the CS to the S and D odors as a function of drug treatment. Since this experiment is largely a replication of previous work in honeybee (Hosler et al., 2000; Stopfer et al., 1997), we used the same 1-tailed paired t-tests, performed in Excel, specifically to compare differences in CRprobability between CS \& S and CS \& D under saline, versus drug (either BMI or PCT) treatments. A significance level of $\mathrm{p}<0.05$ was used for individual comparisons. 
In Experiment 3.2, a number of variables were created to explain the variation in CRprobability. Both treatment and odor were treated as categorical variables indicating which odor was presented (odor) and whether moths were injected with saline or drug (treatment). Drug was also a categorical variable indicating whether BMI or PCT was injected. Concentration was treated as a categorical variable ranging from 0 (air only) to $10 \mu \mathrm{g} / 2 \mu \mathrm{l}$. An additional variable was the CS, a categorical variable that indicated whether an odorant was used as the CS+ or the CS-. Odor pair was a categorical variable indicating which odors were used within a given group; similar (OCT/HEX) or dissimilar (LOL/MES). The main variables of interest however, were the interactions of CS, concentration, treatment and odor pair, which if significant would indicate that the similar versus dissimilar odor pairs produced distinctive differential concentration-response functions in the saline versus drug conditions.

GLM analysis was used to analyze variation in CR-probability as a function of the variables described above. Given the number of post hoc comparisons in this experiment, Tukey's post hoc analysis was implemented to adjust the overall significance level to $\mathrm{p}<0.05$. For Experiment 3.3, GLM was used to analyze variation in CR-probability as a function of concentration, odor, drug and treatment. In this case, the primary effect of interest was the two-way interaction of treatment by concentration. In the final control experiment, 1-tailed paired t-tests were performed in Excel, specifically to compare differences in feeding response between saline and drug (BMI and PCT) treatments. Finally, in all experiments, all possible two and three way interactions were tested. Significance thresholds for all effects in all models were set to $(\mathrm{p}<0.01)$ to reduce experimentwise error rate; $\mathrm{p}<0.05$ was only used in post hoc analysis. 


\section{Results}

\section{Experiment 3.1: Stimulus Generalization}

As mentioned previously, honey bee studies have demonstrated that $\mathrm{GABA}_{\mathrm{A}}$ blockade increases generalization from CS to S but not D odors. The aim of Experiment 3.1 was to confirm these results using Manduca sexta while expanding the number of $\mathrm{GABA}_{\mathrm{A}}$ antagonists and number of odor combinations for which this effect has been documented.

Figure 5 displays the mean CR-probability to the CS, S and D odors as a function of saline and drug injection; specific comparisons are inset to highlight the pattern of effects. In general, saline-treated moths responded significantly more often to CS than they did to S $(\mathrm{p}<0.05)$ and D ( $<<0.01)$. This indicates that they perceived both the S and D odors as distinct from the CS. On the other hand, PCT (Fig. 5a) and BMI (Fig. 5b) treated moths failed to differentiate CS from S. However, by comparison, generalization from the CS to D was equally low for both saline and drug-treated animals. In other words, both groups had a low probability of responding to D relative to the CS ( $\mathrm{p}<0.01$ ) as shown in Figure $5 \mathrm{a}$ and $\mathrm{b}$, indicating that PCT and BMI increased generalization of the CS to S but not to D. These results are consistent with the honeybee study (Stopfer et al., 1997) and suggest that discrimination of closely related odorants is specifically affected by application of $\mathrm{GABA}_{\mathrm{A}}$ antagonists. It should be noted that by comparison with previous results of others, CR-probability elicited by the CS dropped, but insignificantly, in drug treated group relative to the saline group.

\section{Experiment 3.2: Discrimination Thresholds}

Experiment 3.1 results indicate that $\mathrm{GABA}_{\mathrm{A}}$ blockade increased generalization to similar but not dissimilar odors. However, as hypothesized in Figure 1, this does not necessarily mean that discrimination of different odors was unaffected. That is, the dissimilar odor (D) may be affected 
but not necessarily change generalization because, perceptually, it is still sufficiently distinct from the CS. Thus we tested this hypothesis using a discrimination paradigm in Experiment 3.2, whereby moths were differentially conditioned to respond to one odor, CS+ but not the other, CS-. They were then tested with a log step increase in concentrations of the CS beginning with blank and sequentially increasing the concentration pseudo randomly; this allowed us to quantify even subtle effects on discrimination.

The overall statistical model explaining variation in CR-probability for Experiment 3.2 was significant $(\mathrm{p}<0.0001)$. The main effects of CS and concentration were significant $(\mathrm{p}<0.0001)$. Furthermore their interaction was also significant $(\mathrm{p}<0.0001)$ indicating that the effect of reinforcement was concentration-dependent. Figure 6a shows CR-probability as a function of reinforcement across the concentration series and indicates that at lower concentrations there was no difference in CR-probability to the CS+ and CS-. However, as concentration increased, response to CS+ increased significantly over that of CS-.

In addition, we found a significant main effect of treatment $(\mathrm{p}<0.0001)$ but this main effect was also dependent on concentration as indicated by the significant interaction $(\mathrm{p}=0.0006)$. Figure 6b displays the mean CR-probability to odor by stimulus concentration and as a function of whether the moths were injected with drug or saline. Figure $6 \mathrm{~b}$ indicates that moths had a higher probability of eliciting a CR when injected with saline than when injected with BMI or PCT but this was only at concentrations at or above $0.1 \mu \mathrm{g} / 2 \mu \mathrm{l}$. These results suggest that the effect of $\mathrm{GABA}_{\mathrm{A}}$ blockade is in part, a disruption in ability to detect the presence of odor. This effect may also indicate disruption on the moths' ability to respond behaviorally.

There was also a significant three-way interaction of CS, concentration and treatment. Figure 7 displays the mean CR-probability as a function of reinforcement in saline (Fig.7a) versus drug 
(Fig.7b) injected moths. The key trend of interest in these figures is the degree of divergence between CS+ and CS- odors as concentration increases. Notice that in the saline controls, moths begin to significantly differentially respond to the CS+ and CS- at $0.1 \mu \mathrm{g} / 2 \mu \mathrm{l}$, whereas in the drug treated moths a significant differential response does not occur until the highest concentration in the range.

While the above effects indicated that $\mathrm{GABA}_{\mathrm{A}}$ blockade increased the concentration required to discriminate between odors, the main effect of which drug was injected was not significant ( $\mathrm{p}>$ 0.05). This indicates that both BMI and PCT had similar effects in this experiment. In fact there were also no significant interactions with the drug effect and any other main effect indicating that BMI and PCT had essentially the same impact on olfactory function.

Finally, neither the main effect of the odor pair used, nor any of its interactions with the other variables in the model were significant ( $p>0.05$ ). In particular, the lack of a significant four-way interaction of CS by concentration by treatment by odor pair indicates that the similarity of odors in a pair has no impact on the magnitude of the effect on discrimination measures. This lack of effect can be visualized in Figure 8. Here the three-way interaction of CS by concentration by treatment was further broken down by the closely related (Fig. 8a and 8a $a_{i i}$ ) versus unrelated (Fig. $8 b_{i}$ and $\left.8 b_{i i}\right)$ odor pairs. Notice that in both cases where saline was injected, the discrimination thresholds for these pairs is at the same concentration, $0.1 \mu \mathrm{g} / \mu \mathrm{l}$, though the dissimilar odors diverge more rapidly. This represents a two-log step lower discrimination threshold than when BMI was injected. By comparison, similar results were obtained particularly for unrelated odors (LOL/MES) when PCT was injected (Fig. 9). Based on the expectations set up from Experiment 3.1 results, the effect of drug treatment on discrimination thresholds should have been greater for the closely related odors. Unlike Experiment 3.1, however, these results indicate that GABA 
blockade impaired discrimination of similar and dissimilar odors equally. These results suggest that a generalization paradigm (as used in Experiment 3.1), incorrectly quantifies the effect of $\mathrm{GABA}_{\mathrm{A}}$ disruption on discrimination of different odors.

\section{Experiment 3.3: Detection Thresholds}

The significant interaction of concentration by treatment in Experiment 3.2 (see Fig. 6b) suggested that detection thresholds may have increased. This led us to hypothesize that the underlying drug treatment-related increased discrimination thresholds were mediated by a disruption in ability to detect odor. Previous studies have established that discrimination thresholds occur at least one order of magnitude above detection thresholds (Daly et al., in press; Daly et al in review). Thus, if detection thresholds increased as a function of $\mathrm{GABA}_{\mathrm{A}}$ blockade, then it stands to reason that discrimination thresholds should increase as well. We, therefore, quantified the effects of BMI and PCT on detection thresholds.

The overall statistical model explaining variation in CR-probability for Experiment 3.3 was significant $(\mathrm{p}<0.0001)$. We found that there were significant main effects of concentration and treatment $(p<0.0001)$ but no significant effect of which drug treatment was used $(p>0.01)$. Importantly, the interaction of concentration by treatment was also significant $(\mathrm{p}<0.0001)$. Figure 10a displays the mean CR-probability as a function of concentration for both saline and drug treated moths. What Figure 10a indicates is that across all odors BMI and PCT treated moths produced higher detection thresholds than saline-treated moths, suggesting that the loss of discrimination ability observed in Experiment 3.2 is likely due to a general impairment of the moth to detect odor. This indicates that for all four odors used, both BMI and PCT had similar effects.

A final possibility was that this drug-induced increase in detection threshold was attributable to changes in ability to produce a behavioral response. To test this hypothesis, we simply provided 
sucrose to each moth's proboscis immediately following the final odor test. If moths were less able to respond to sucrose as well, this would suggest that underlying the increased detection threshold measure was an inability to produce the behavior and thus not attributable to changes in detection per se. Figure 10b displays the mean unconditioned response probability to sucrose presentation upon the proboscis. T-tests of differences between the saline and each drug treatment indicate that PCT and BMI had no significant effect on the moths' ability to feed ( $\mathrm{p}>0.05$ ). 


\section{Discussion}

GABA blockade increases generalization to similar but not dissimilar odors: A generalization paradigm

In agreement with previous studies, we find that generalization to a conditioned response is increased to molecularly closely related odors only when GABA blockers are applied to the AL. In a previous report by Stopfer et al., (1997), topical application of PCT to honeybee ALs prior to conditioning produced a disrupted memory template of the CS odor. This resulted in increased generalization of closely related odors. In another study by Hosler et al., (2000), it was established that PCT can be applied either before conditioning or before testing and the same results occur. This suggests in both cases (whether the memory template is accurate but the test odor images are disrupted or visa versa) the end result is the same; fine odor discrimination is disrupted. Theoretically, however, a generalization paradigm tests whether stimulus $\mathrm{X}$ is perceived to be like stimulus Y. On the other hand, Discrimination tasks test whether the test subject can tell the difference between $\mathrm{X}$ and $\mathrm{Y}$. Thus we proposed that the generalization method yields the specific pattern of increased generalization from the CS to the S odor simply because it is a closely related stimulus at the outset; generalization from the CS to the D would not necessarily be affected in the paradigm even if the animals’ ability to discriminate CS from D was affected.

GABA blockade impairs discrimination of similar and dissimilar odors: A discrimination learning paradigm

We have previously established methods for psychophysically quantifying discrimination thresholds in $M$. sexta as well as differential concentration-response functions, which describe the rate at which the differential response to the CS+ as CS- diverge as a function of increasing concentration (Daly et al., in review). Using this method we can determine with relative precision 
the degree to which moths can discriminate between odors of a pair. Consistent with our previous findings, we observe that the ability to discriminate only increases with increasing concentration. When we disrupted normal $\mathrm{GABA}_{\mathrm{A}}$ function, however, we observed that discrimination of both closely related and unrelated odors were equally disrupted. This was evidenced by a two log-step increase in the concentration necessary to elicit a differential response to the CS+ and CS- for both similar and dissimilar odor pairs. These findings are in contrast to those observed using the generalization paradigm and suggest that the effect of $\mathrm{GABA}_{\mathrm{A}}$ blockade is more profound than previously described.

\section{GABA blockade increases detection thresholds}

Finally, underlying the general loss of ability to discriminate between odors, we observe that $\mathrm{GABA}_{\mathrm{A}}$ disruption in the $\mathrm{AL}$ contributes to a loss of ability to detect the presence of an odor stimulus. We have shown in a previous report that detection thresholds correlate well with matched physiological measures (Daly et al., in press). By implementing these detection threshold methods in drug treated versus control experiments, we were able to establish that that $\mathrm{GABA}_{\mathrm{A}}$ disruption indeed affects detection of odor. Obviously, a prerequisite to discrimination of any two stimuli is that they are detectable in the first place. Typically, moths detected the odors used herein at concentrations one or more orders of magnitude below what is necessary for discrimination.

Subsequent assessment of moths' ability to elicit an unconditioned response to the sucrose solution strongly suggests that the increased detection thresholds cannot be attributed to an inability to respond with a behavioral response. That is, the sensory-motor circuitry responsible for driving the activation and maintenance of feeding behavior, at least in response to gustatory input, remains intact. Furthermore, the fact that higher concentrations of odor stimuli were able to elicit a conditioned response further suggest that the sensory-motor circuitry involved in driving the 
conditioned olfactory-mediated responses was at least partially functional. Thus we conclude that the main effect of PCT and BMI application was to disrupt qualities of AL output that related directly to signal detection, which we presume forms the basis of signal discrimination. 


\section{CONCLUSION}

The overall goal of this study was to psychophysically quantify odor detection and discrimination thresholds and characterize the change in these measures as a function of increasing stimulus concentration and blockade of $\mathrm{GABA}_{\mathrm{A}}$. We have shown that detection and discrimination thresholds and concentration-response functions can be generated using a Pavlovian paradigm and that this general method correlates well with matched physiological measures (Daly and Smith, 2000; Daly et al., 2001a,b; Daly et al., in press; Daly at al., in submission). In addition, our results clearly indicated that odor detection and discrimination thresholds varied widely with different odors. However, we see that discrimination, though a unique process, must be dependent on detection. We make this conclusion based on the observation that moths detect these odors at concentrations 1 or more orders of magnitude below what is necessary for discrimination (Daly at al., in submission).

Further more, results of our third study imply that the physiological effects of $\mathrm{GABA}_{\mathrm{A}}$ blockade are more functionally systemic than previously reported (Stopfer et al., 1997). Temporal models of olfactory encoding have suggested that $\mathrm{GABA}_{\mathrm{A}}$ blockade specifically mediates the abolition of local field potential oscillations within the AL leaving slow patterns of output intact (Wehr and Laurent, 1996); this in turn desynchronizes distributed and transiently synchronized neural assemblies. The result of this is that discrimination of odors, which under normal conditions activate the same "glomerular map," is disrupted. This model of fine odor discrimination is not supported by the data presented herein. Therefore, we conclude that the psychopharmacological basis of this transient oscillatory model of odor encoding, which used a 
generalization paradigm that was inadequate to quantify effects of $\mathrm{GABA}_{\mathrm{A}}$ blockade on discrimination of molecularly different odors or stimulus detection, was insufficient. 


\section{APPENDIX A}

\section{Figures}

Figure 1. Generalization gradient showing the response probability of a conditioned odor (CS), similar odor (S) and dissimilar odor (D). $\mathrm{S}_{\mathrm{g}}$ and $\mathrm{D}_{\mathrm{g}}$ represent theoretical positions of $\mathrm{S}$ and $\mathrm{D}$ where discrimination has been impaired by GABA blockade. Numerals 1 and 2 indicate the magnitude of the theoretical effect of drug treatment for S (1) and D (2) odors relative to the CS. Numeral 3 indicates the theoretical increase in CR-probability of odor S, which suggests an increase in generalization. Note that the prediction of this model is that while the perceptual similarity of CS and $\mathrm{D}$ has been affected, there is no increase in generalization.

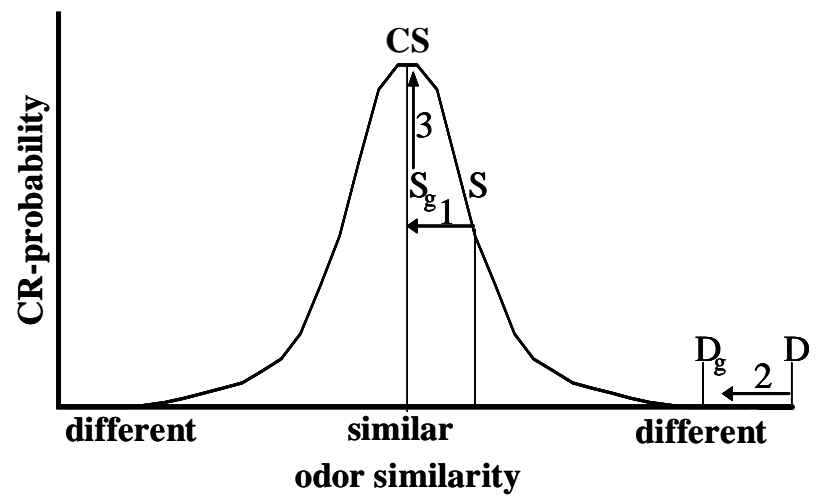


Figure 2. Mean CR-probability as a function of odor (A) and concentration (B). Inset letters indicate significant differences between odors with different letters $(p<0.01)$ and error bars indicate standard error. Note that the CR-probability increases with concentration across all odors used.

A.

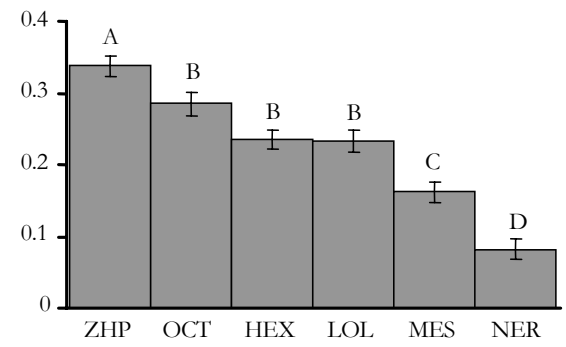

B.

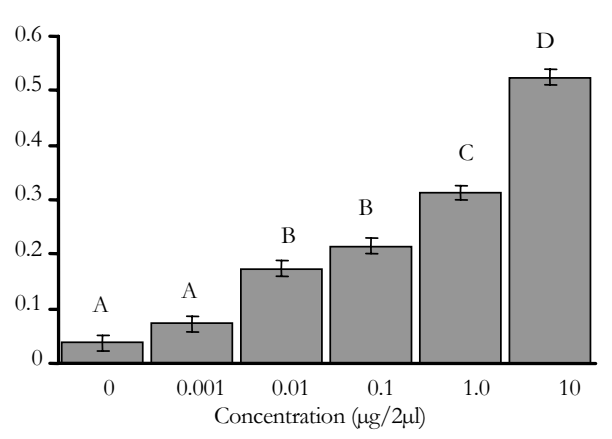


Figure 3. Mean CR-probability as a function of concentration by odor. Error bars are standard errors. Inset regression lines are based on a third order polynomial function to model increasing response probability as a function of concentration. Inset $\mathrm{R}^{2}$ refers to the overall fit of the third order polynomial to the mean responses by concentration. Asterisk indicates the lowest concentration at which the mean CR-probability was statistically higher than that elicited by a blank. A. Cis-3-hexenyl proprionate. B. 2-octanone. C. 2-hexanone. D. +/-linalool. E. Methylsalicylate. F. Cis-nerolidol.

A.
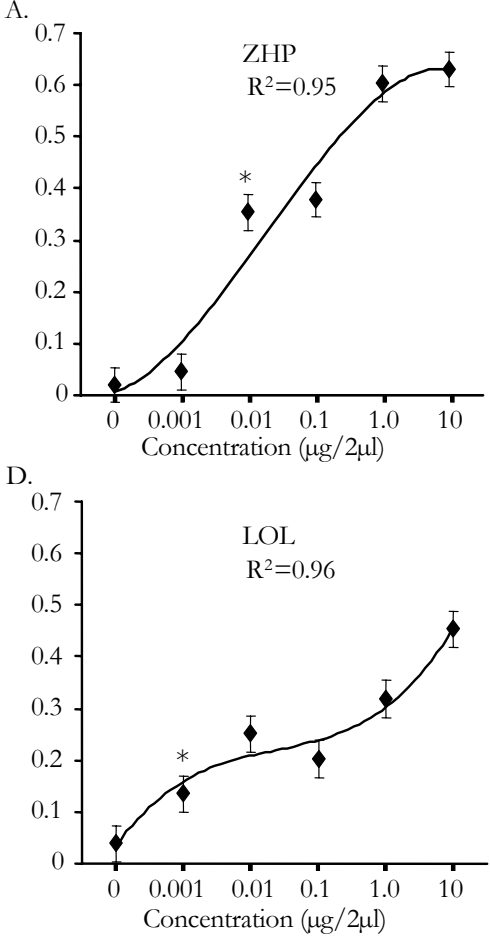

B.

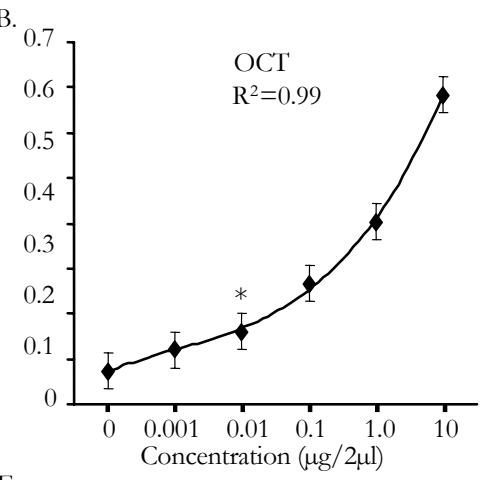

E.

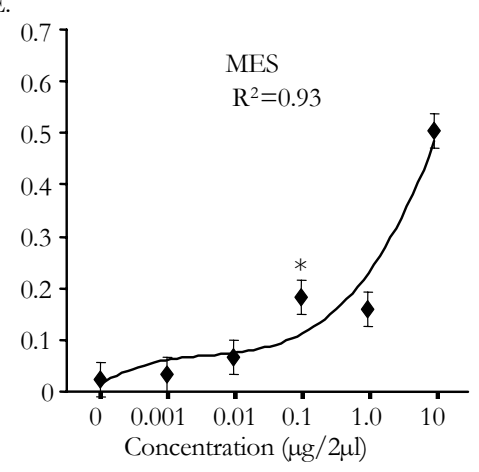

C.
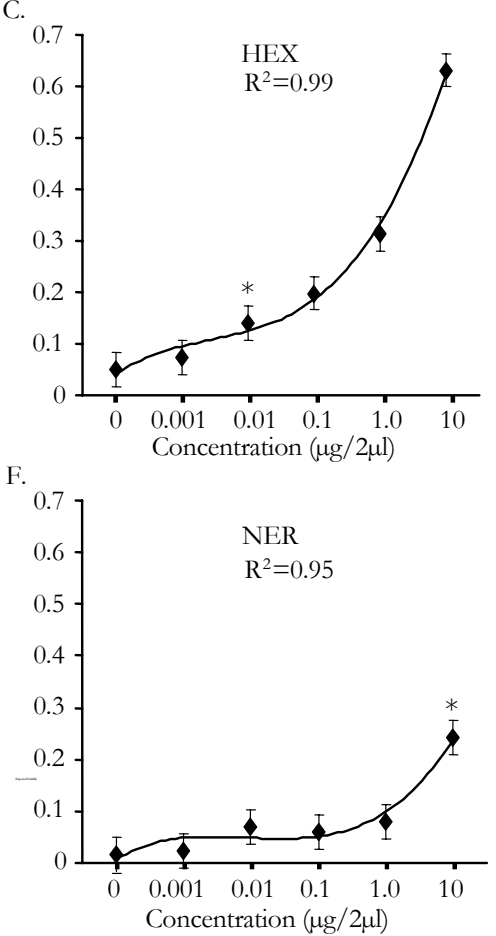
Figure 4. Mean CR-probability as a function of the three-way interaction of CS by concentration by odor pair. Panels A-E breaks this interaction down by odor pair and CS so that the symmetry within individual odor pairs can be assessed. For example A displays the differential responses to the CS+ and CS- for HEX and LOL when LOL is the CS+ $\left(\mathrm{A}_{\mathrm{i}}\right)$ and the CS- $\left(\mathrm{A}_{\mathrm{ii}}\right)$. Discrimination thresholds are denoted by an asterisk $(* \mathrm{p}<0.05)$.

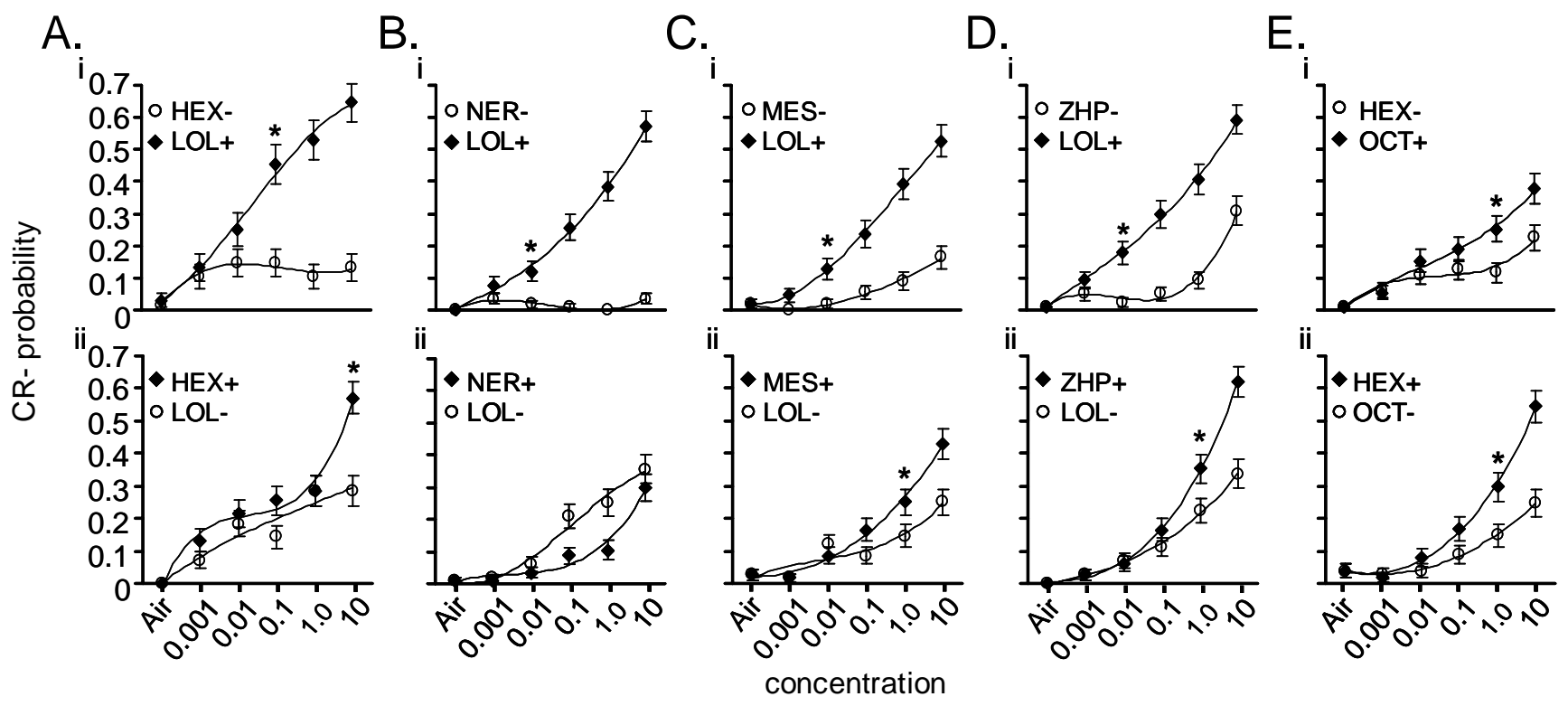


Figure 5. Mean CR-probability as a function of odor (CS, S, D), treatment (saline, drug) and drug group (BMI and PCT). Results of specific post-hoc comparisons using 1-tailed paired t-tests are inset and indicated by (-*-) for significant effects and (-NS-) for non-significant effects. Error bars indicate standard error. In both $\mathrm{A}$ and $\mathrm{B}$, the response probability for saline-treated moths decreases as the similarity of the test odor to CS decreases. Note that difference in CR-probability for only CS to S becomes insignificant as a result of PCT and BMI treatments respectively

A. PCT

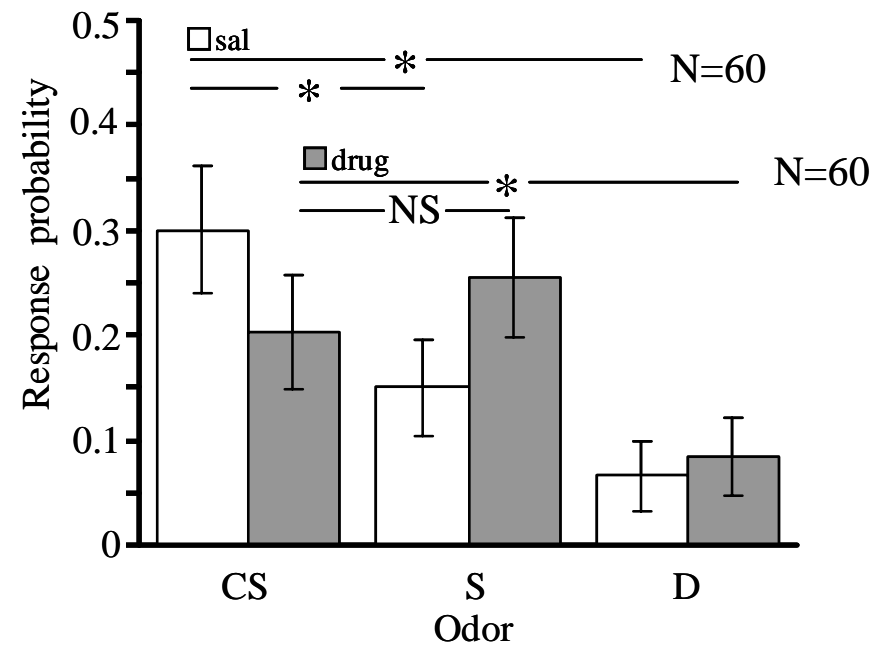

B. BMI

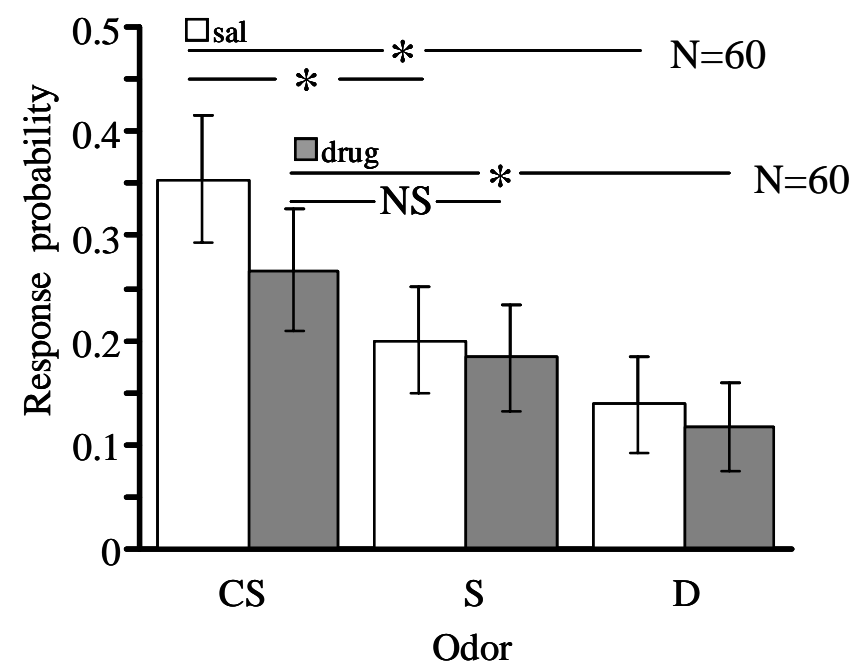


Figure 6. Mean CR-probability as a function of the two-way interactions of concentration by CS (A) and treatment (B). A. Data is averaged across treatment (saline and drug) and drug group (BMI and PCT). As concentration increases, there is an increase in CR-probability to the CS+ as relative to the CS-. B. Data is averaged across CS (CS+ and CS-) and drug group (BMI and PCT). Significant post-hoc comparisons are inset $(*)$ and error bars indicate standard error. Note that as concentration increases, saline-treated moths elicit a significantly higher CR-probability of response than drug-treated moths.

A.

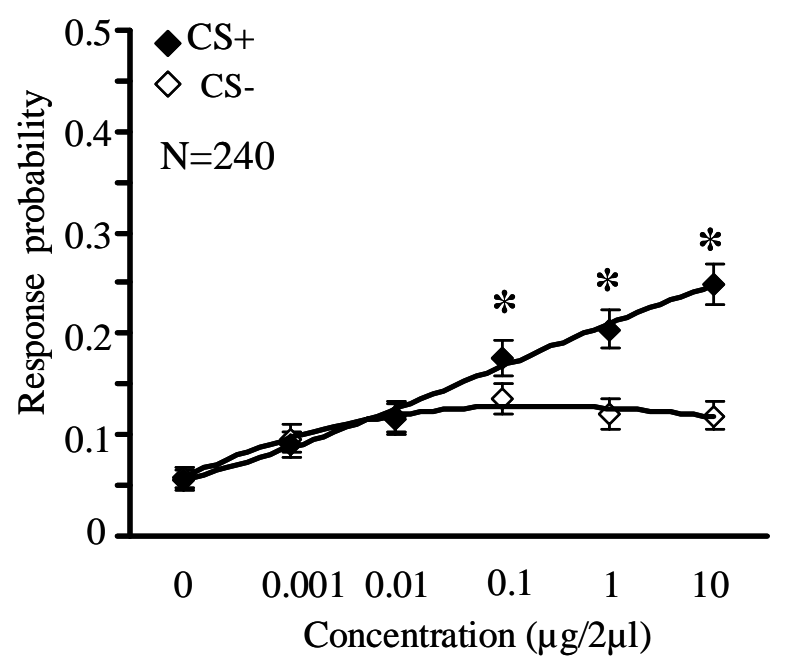

B.

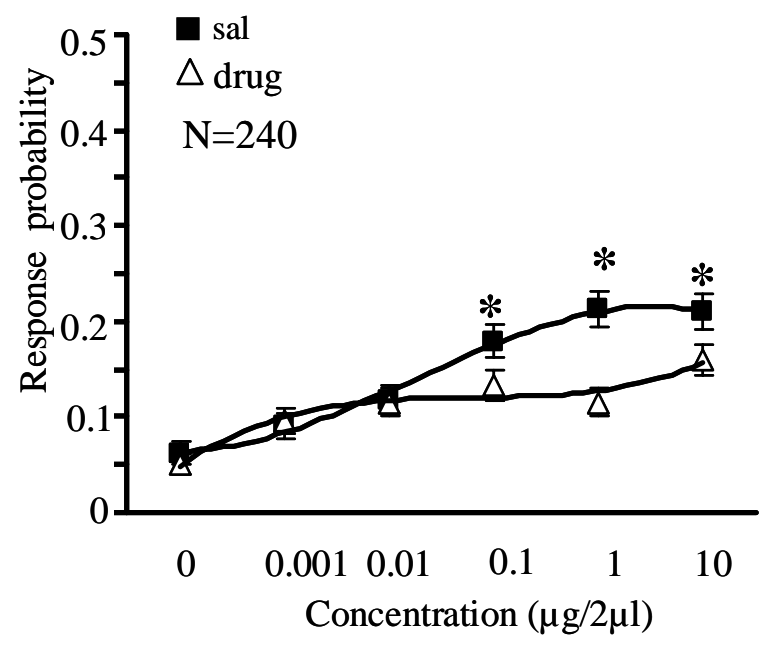


Figure 7. Mean CR-probability as a function of the three-way interaction of CS by concentration by drug treatment. These data are collapsed across drug group (BMI and PCT) and broken into saline (A) and drug injected (B). Significant post-hoc comparisons are inset (*) and error bars indicate standard error. Note the distinct divergence of CS+ and CS- beginning at $0.1 \mu \mathrm{g} / \mu \mathrm{l}$ in saline-treated moths; this divergence does not occur until the highest concentration in drug-treated moths.

\section{A. SAL}

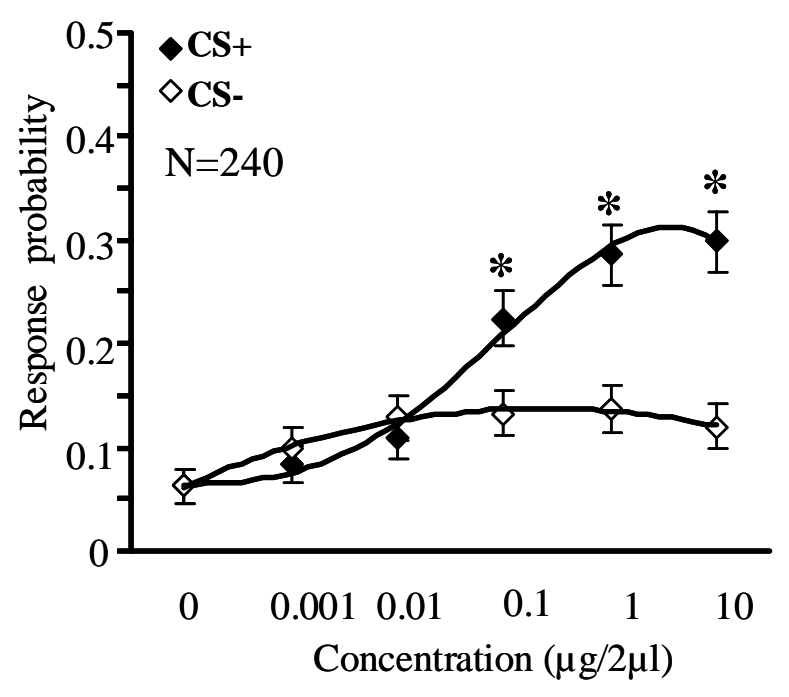

B. DRUG

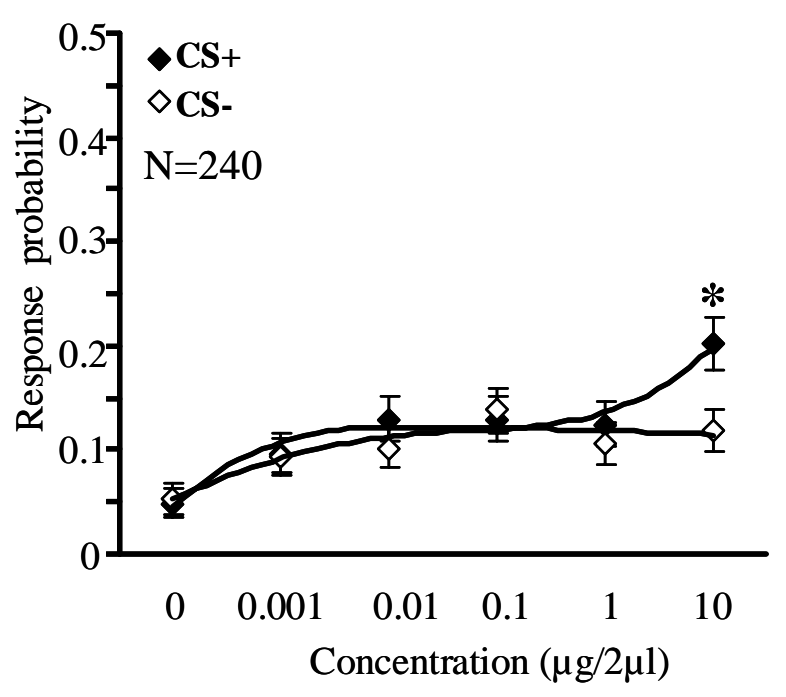


Figure 8. Mean CR-probability as a function of the three-way interaction of CS by concentration by treatment for saline and BMI. These data are further broken into two panels representing similar (Ai and Aii; OCT/HEX) and dissimilar (Bi and Bii; LOL/MES) odors. Discrimination thresholds are indicated by $\left(^{*}\right)$ and error bars indicate standard error. Again, there are distinct divergences of $\mathrm{CS}+$ and CS- that begin at $0.1 \mu \mathrm{g} / \mu \mathrm{l}$ for saline-treated moths in both $\mathrm{Ai}$ and $\mathrm{Bi}$ and the drug related effect on this divergence is equal for both similar and dissimilar odor pairs (Aii and Bii).

Ai. SAL

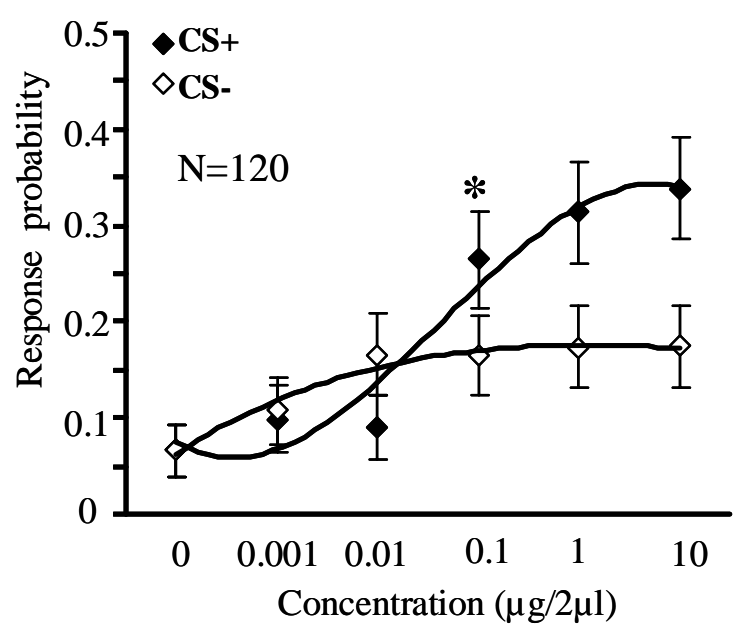

Bi. SAL

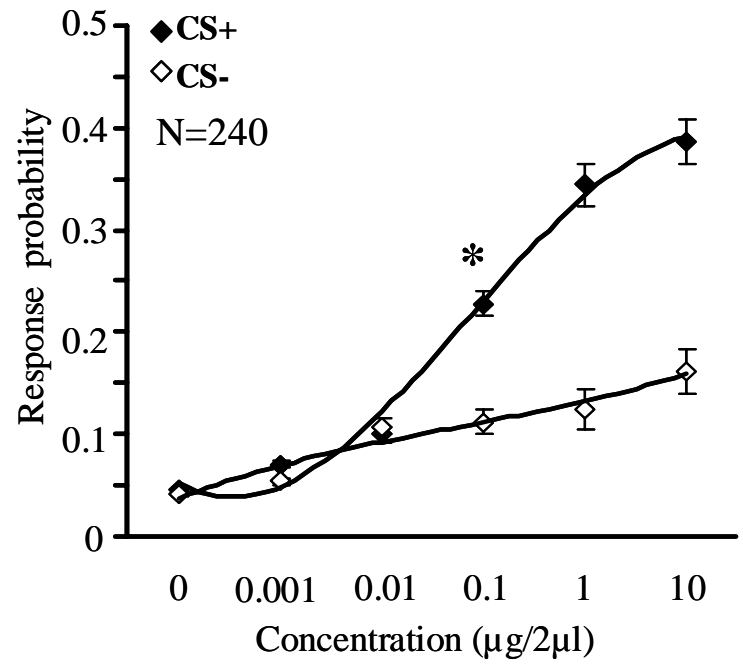

Aii. DRUG

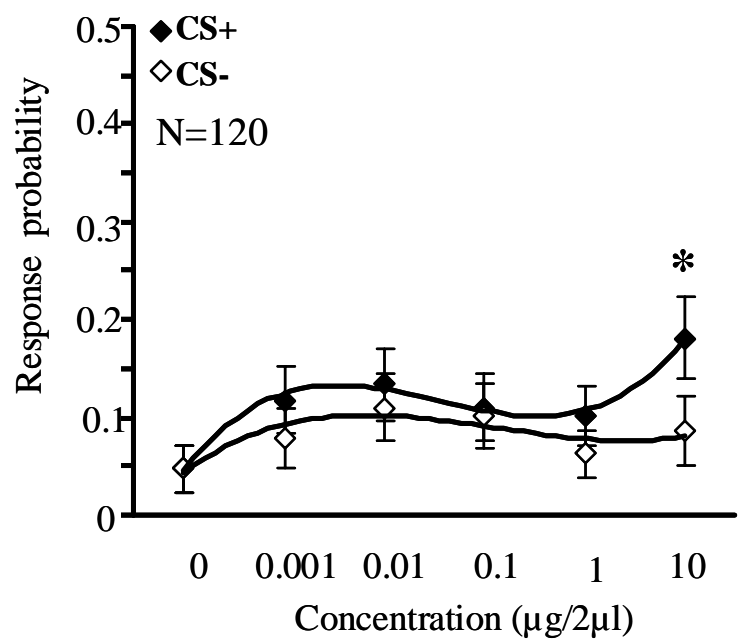

Bii. DRUG

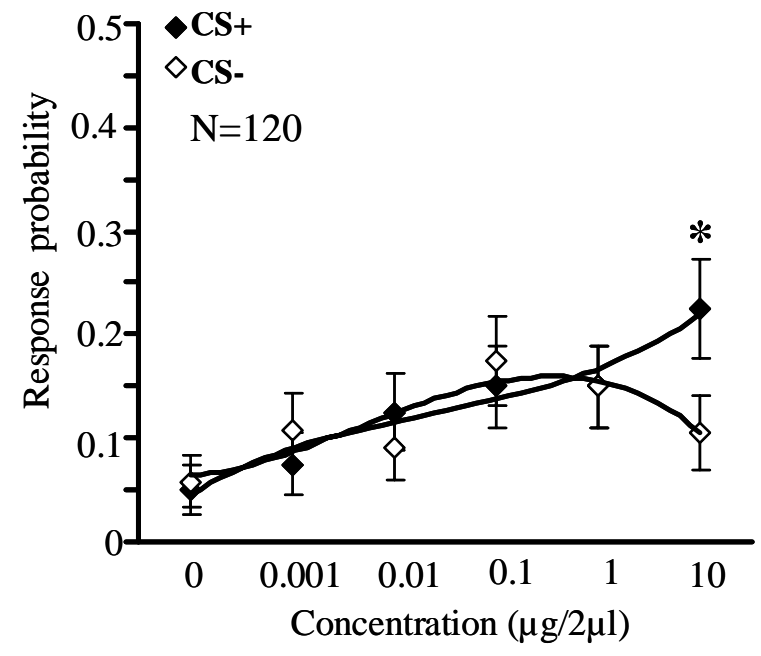


Figure 9. Mean CR-probability as a function of the two-way interaction of CS by concentration for dissimilar odors (LOL/MES) under PCT treatment. The discrimination threshold is indicated by $\left(^{*}\right.$ ) and error bars indicate standard error. These results can be compared to saline control data presented in Figure $5 b_{i}$. Note the lack of divergence of CS+ and CS- polynomials for PCT-treated moths that is consistent with BMI treatment.

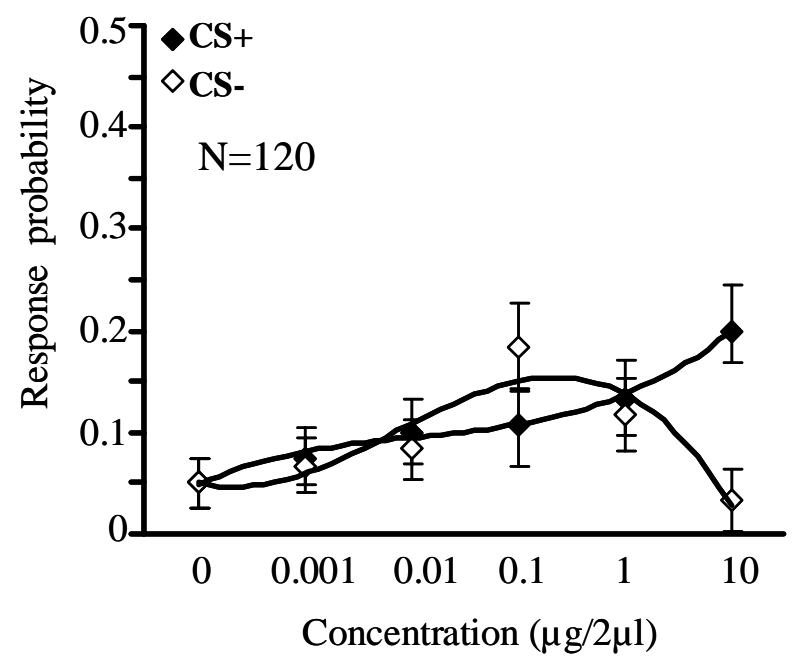


Figure 10. (A) Mean CR-probability as a function of the two-way interaction of concentration by treatment. These data are collapsed across drug group (BMI and PCT) and odor (OCT, HEX, LOL and MES). Significant post-hoc comparisons are inset (*) and error bars indicate standard error. We display results averaged across odors because the pattern was the same in all odor comparisons. As concentration increases, the probability of response increases in saline but not drug treated moths. (B) Mean post-test feeding response probability upon presentation of sucrose solution to the proboscis. Results of specific post-hoc comparisons using 1-tailed paired t-tests are inset and indicated by (NS) for non-significant effects. Error bars indicate standard error.
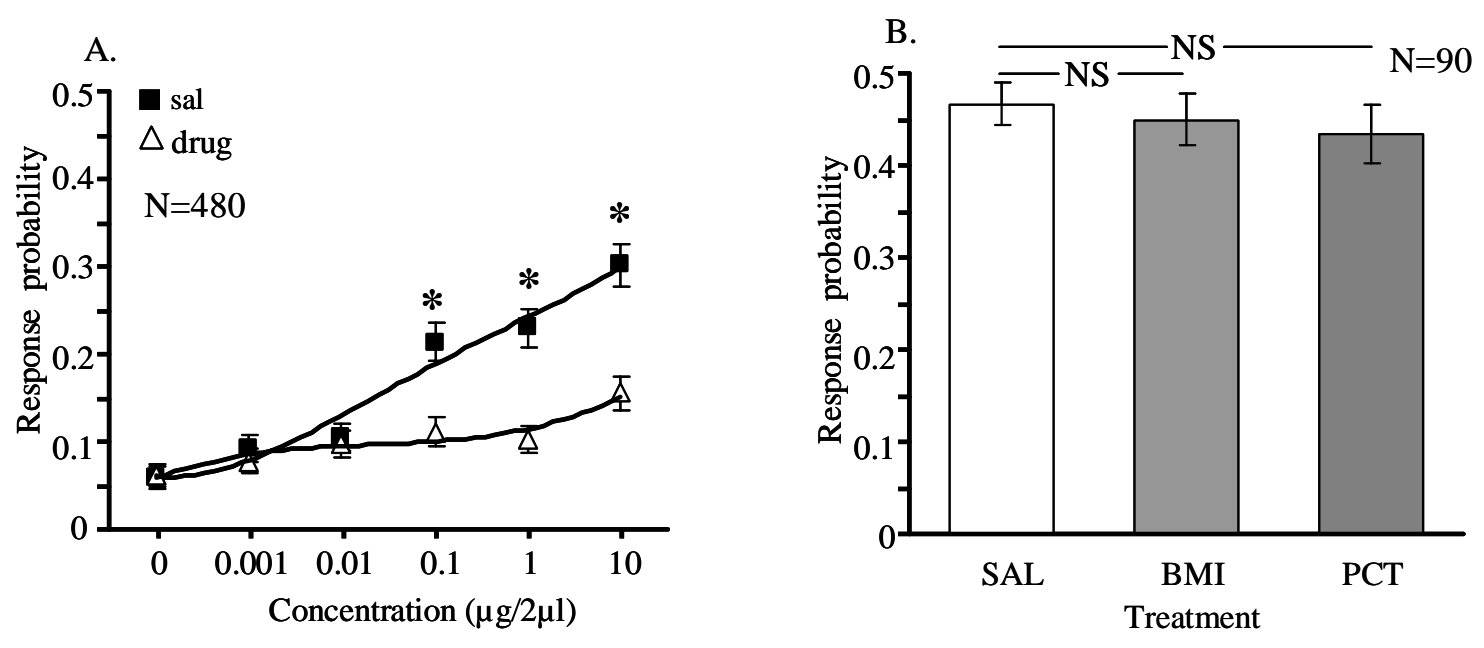


\section{References}

Balkenius, A. and Kelber, A. (2006). Colour preferences influences odour learning in the hawkmoth, Macroglossum stellatarum. Naturwissenschaften 93, 255-8.

Bhagavan, S. and Smith, B. H. (1997). Olfactory conditioning in the honey bee, Apis mellifera: effects of odor intensity. Physiol Behav 61, 107-17.

Christensen, T. A., Waldrop, B. R., Harrow, I. D. and Hildebrand, J. G. (1993). Local interneurons and information processing in the olfactory glomeruli of the moth Manduca sexta. J Comp Physiol [A] 173, 385-99.

Christensen, T. A., Waldrop, B. R. and Hildebrand, J. G. (1998). Multitasking in the olfactory system: context-dependent responses to odors reveal dual GABA-regulated coding mechanisms in single olfactory projection neurons. $J$ Neurosci 18, 5999-6008.

Cleland, T. A., Morse, A., Yue, E. L. and Linster, C. (2002). Behavioral models of odor similarity. Behav Neurosci 116, 222-31.

Cleland, T. A. and Narla, V. A. (2003). Intensity modulation of olfactory acuity. Behav Neurosci 117, 1434-40.

Daly, K. C., Chandra, S., Durtschi, M. L. and Smith, B. H. (2001a). The generalization of an olfactory-based conditioned response reveals unique but overlapping odour representations in the moth Manduca sexta. J Exp Biol 204, 3085-95.

Daly, K. C., Durtschi, M. L. and Smith, B. H. (2001b). Olfactory-based discrimination learning in the moth, Manduca sexta. J Insect Physiol 47, 375-84.

Daly, K. C. and Smith, B. H. (2000). Associative olfactory learning in the moth Manduca sexta. J Exp Biol 203, 2025-38.

Daly, K. C., Wright, G. A. and Smith, B. H. (2004). Molecular features of odorants systematically influence slow temporal responses across clusters of coordinated antennal lobe units in the moth Manduca sexta. J Neurophysiol 92, 236-54.

Eaton, J. L. (1971). Morphology of the head and thorax of the adult tobacco hornworm Manduca sexta (Lepidoptera: Sphingidae). 1. Skeleton muscles. Annals of the Entomological Society of America. 64, 437-445.

Fan, R. J. and Hansson, B. S. (2001). Olfactory discrimination conditioning in the moth Spodoptera littoralis. Physiol Behav 72, 159-65.

Hildebrand, J. G. and Shepherd, G. M. (1997). Mechanisms of olfactory discrimination: converging evidence for common principles across phyla. Annu Rev Neurosci 20, 595-631.

Hosler, J. S., Buxton, K. L. and Smith, B. H. (2000). Impairment of olfactory discrimination by blockade of GABA and nitric oxide activity in the honey bee antennal lobes. Behav Neurosci 114, 51425.

Laska, M., Galizia, C. G., Giurfa, M. and Menzel, R. (1999). Olfactory discrimination ability and odor structure-activity relationships in honeybees. Chem Senses 24, 429-38.

Laurent, G. and Davidowitz, H. (1994). Encoding of Olfactory Information with Oscillating Neural Assemblies. Science 265, 1872-1875.

Laurent, G., Stopfer, M., Friedrich, R. W., Rabinovich, M. I., Volkovskii, A. and Abarbanel, H. D. I. (2001). Odor encoding as an active, dynamical process: Experiments, computation, and theory. Annual Review of Neuroscience 24, 263-297.

Leitch, B. and Laurent, G. (1996). GABAergic synapses in the antennal lobe and mushroom body of the locust olfactory system. Journal of Comparative Neurology 372, 487-514. 
Matsumoto, S. G. and Hildebrand, J. G. (1981). Olfactory mechanisms in the moth manduca sexta: Response characteristics and morphology of central neurons in the antennal lobe. . Proceedings of the Royal Society London B 213, 249-277.

Sachse, S. and Galizia, C. G. (2002). Role of inhibition for temporal and spatial odor representation in olfactory output neurons: a calcium imaging study. J Neurophysiol 87, 1106-17.

Sakura, M., Okada, R. and Mizunami, M. (2002). Olfactory discrimination of structurally similar alcohols by cockroaches. J Comp Physiol A Neuroethol Sens Neural Behav Physiol 188, 787-97.

Shipley, M. T. and Ennis, M. (1996). Functional organization of olfactory system. J Neurobiol 30, 12376.

Skiri, H. T., Stranden, M., Sandoz, J. C., Menzel, R. and Mustaparta, H. (2005). Associative learning of plant odorants activating the same or different receptor neurons in the moth Heliothis virescens. J Exp Biol 208, 787-96.

Stopfer, M., Bhagavan, S., Smith, B. H. and Laurent, G. (1997). Impaired odour discrimination on desynchronization of odour-encoding neural assemblies. Nature 390, 70-74.

Waldrop, B., Christensen, T. A. and Hildebrand, J. G. (1987). GABA-mediated synaptic inhibition of projection neurons in the antennal lobes of the sphinx moth, Manduca sexta. J Comp Physiol [A] 161, 23-32.

Wang, Y., Wright, N. J., Guo, H., Xie, Z., Svoboda, K., Malinow, R., Smith, D. P. and Zhong, Y. (2001). Genetic manipulation of the odor-evoked distributed neural activity in the Drosophila mushroom body. Neuron 29, 267-76.

Wehr, M. and Laurent, G. (1996). Odour encoding by temporal sequences of firing in oscillating neural assemblies. Nature 384, 162-6.

Wright, G. A., Lutmerding, A., Dudareva, N. and Smith, B. H. (2005). Intensity and the ratios of compounds in the scent of snapdragon flowers affect scent discrimination by honeybees (Apis mellifera). J Comp Physiol A Neuroethol Sens Neural Behav Physiol 191, 105-14.

Wright, G. A., Skinner, B. D. and Smith, B. H. (2002). Ability of honeybee, Apis mellifera, to detect and discriminate odors of varieties of canola (Brassica rapa and Brassica napus) and snapdragon flowers (Antirrhinum majus). J Chem Ecol 28, 721-40. 
CURRICULUM VITAE

\section{Esther Kananu Mwilaria}

Contact Address:

10B Chalfant Alley

Morgantown, WV 26505
PH: 304.685.8224

EM1: emwilari@mix.wvu.edu

EM2: kemwilaria@hotmail.com

\section{Education:}

- $\quad$ M.S. Biology. West Virginia University, Morgantown, WV (2007). GPA 3.85.

- B.A. Biology. Washington and Jefferson College, Washington, PA (2004). GPA 3.38.

\section{Publications:}

- Daly K. C., Carrell L. A., Mwilaria E. K. Detection versus perception: Physiological and behavioral analysis of olfactory sensitivity in the moth, Manduca sexta (In press).

- Daly K. C., Carrell L. A., Mwilaria E. K. The effect of stimulus intensity on discrimination learning and performance in the moth Manduca sexta (In review).

- Mwilaria E. K. and Daly K. C. Disruption of GABA in the insect antennal lobe impairs discrimination by increasing detection thresholds (In review).

\section{Presentations:}

\section{Posters/Orals}

The effect of stimulus intensity on discrimination learning and performance in the moth Manduca sexta. AChemS 28th Annual Meeting, Sarasota FL (April, 2006)

Disruption of GABA in the insect antennal lobe impairs discrimination by increasing detection thresholds. AChemS 29th Annual Meeting, Sarasota FL (April, 2007)

\section{Teaching Experience}

Biology 115. Principles of Biology Lab: 2 sections

\section{Health Related Experience}

- $\quad$ Public Health Intern, Jackson WY (May-July, 2002)

- $\quad$ Health Care Assistant, Morgantown, WV (Jan 2004 - Jan 2005) 


\section{Training and Certifications}

First-Aid, CPR, MANDT and OSHA 2004-2007

\section{Volunteerism:}

- $\quad$ Presbyterian Nursing home volunteer, Washington, PA (2002-2003)

- United Nations AIDS awareness Volunteer, Kenya (July-August 2002)

- After-school Program Assistant, Washington, DC (2001-2002)

\section{Awards:}

National Academic Award for Minority Students; Washington and Jefferson College, Washington, PA (2003).

\section{References:}

Dr. Buba Misawa

Professor, Political Science

60 South Lincoln Street

Washington, PA 15301

PH: (724) 503-1070

EM: bmisawa@washjeff.edu

\section{Degree advisor contact information:}

M.S.

Dr. Kevin Daly

Assistant Professor

Department of Biology

P.O. Box 6057

West Virginia University

Morgantown, WV 26506

PH: 304.293.5201

extensions: Office: 31465; Lab: 31467

FX: 304.293.6363

EM:kevin.daly@mail.wvu.edu
Grace Atebe

Student Advisor

Pierpont Apts

P.O. Box 6430

Morgantown, WV 26506

PH: (304) 293-9737 ext 19040

EM: grace.atebe@mail.wvu.edu
B.A.

Dr. Dennis Trelka

Washington and Jefferson College

60 South Lincoln Street

Washington, Pa 15301

PH: 724-503-1001, Ext. 6121

EM: dtrelka@washjeff.edu 